\title{
A phenomenological model for chemico-mechanically induced cell shape changes during migration and cell-cell contacts
}

\author{
F. J. Vermolen • A. Gefen
}

Received: 22 November 2011 / Accepted: 26 April 2012 / Published online: 24 May 2012

(C) The Author(s) 2012. This article is published with open access at Springerlink.com

\begin{abstract}
A phenomenological model for the evolution of shape transition of cells is considered. These transitions are determined by the emission of growth-factors, as well as mechanical interaction if cells are subjected to hard impingement. The originality of this model necessitates a formal treatment of the mathematical model, as well as the presentation of elementary cases in order to illustrate the consistence of the model. We will also show some small-scale relevant applications.
\end{abstract}

Keywords Cell deformation - Chemotaxis .

Phenomenological modeling

\section{Introduction}

Cells are known to migrate under the influence of chemical factors, such as growth factors or repelling agents as well as due to presence of other cells. During cellular motion, the cell shape takes the form of elongated oval geometry, whereas resting cells present typically bi-polar or multi-polar shapes. In order to understand biological processes like wound healing, tumor growth, organ development, or infection by invading bacteria or other invading colonies, it is crucially important to gain understanding on a cellular level, since such understanding may lead to development of improved medical treatments. To accomplish a high level of understanding, the

F. J. Vermolen $(\varangle)$

Delft Institute of Applied Mathematics, Delft University

of Technology, Delft, The Netherlands

e-mail: F.J.Vermolen@tudelft.nl

A. Gefen

Department of Biomedical Engineering, Tel Aviv University, 69978 Tel Aviv, Israel

e-mail: gefen@eng.tau.ac.il relation between various biologically important parameters needs to be scrutinized. For this reason, mathematical relations between these various parameters are indispensable, and hence, mathematical modeling plays a crucial role. In confluent cultures, cells typically form cobblestone-shaped structures. The polygonal character of the confluent cells depends on the culture density as well as on any mechanical stimuli (such as shear flow) which might be present.

For the modeling of processes like wound healing, tumor growth or bone-ingrowth, a lot of mathematical studies have been devoted to continuum based models, which are formulated in terms of partial differential equations (PDEs). In the mathematical literature, one encounters simplified models for which analytic solutions are constructed, whereas for more complicated modeling studies, finite-element (or any other discretization method) simulators are developed. These models are useful on a tissue scale, where cells are not tracked, but rather cell densities are modeled. Some of these PDE models track the interface between for instance wound or tumor and the undamaged tissue, explicitly by incorporating a moving boundary problem (Adam 1999; Mi et al. 2007; Arciero et al. 2011; Schugart et al. 2008; Xue et al. 2009; Javierre et al. 2008; Vermolen and Javierre 2009). Further, (Armstrong et al. 2006; Gerisch and Chaplain 2006), some studies were performed based on the use of non-local (integral) terms in PDE's to simulate interfaces between different cell-types. Other PDE models simulate the transition between wound or tumor and undamaged tissue as more gradual; however, there still remains a relatively sharp transition between the two states (wound/tumor and undamaged tissue) (Sherratt and Murray 1991; Gaffney et al. 2002; Vermolen 2008). Mechanical issues are also often dealt with by the use of visco-(hyper)elastic models (Murray 2004; Olsen et al. 1995; Javierre et al. 2009; Vermolen and Javierre 2010, 2011). However, these PDE models are not capable of simulating 
processes of a cellular scale. We finally remark that some reviews on PDE modeling of biological processes were written by Geris et al. (2010), Geris et al. (2009), Baker et al. (2008), Murray (2004), where both mechanical balances and transport through random walk, tenso-taxis and chemo-taxis were taken into account.

Since cell-level models can provide information about processes that take place in individual cells, these models are critically important. Some research effort should therefore be devoted to the transition between the models that were constructed at these two scales. In the experimental research communities, there is also a clear separation between these investigators working on cell-cultures and tissue scale in terms of in vitro and in vivo experiments, respectively. Cellbased models involve the cellular-Potts models, which fall within the class of cellular automata models. These models (initiated by Graner and Glazier 1992) are based on principles from theoretical physics in the sense that a virtual energy is minimized using a Monte-Carlo like algorithm to allow a certain degree of randomness. Furthermore, these models are lattice-based, which means that cells are allowed to take a discrete set of positions, classically distributed in a Cartesian way, within a certain domain of computation. Using these models, one succeeds in modeling the complicated process of angiogenesis (Merks and Koolwijk 2009).

An alternative formalism (Vermolen and Gefen 2012) is developed to simulate the motion of cells on a planar substrate, in which cells communicate and are attracted toward each other by the pulling forces that they exhibit and sense. This model is a cell-based formalism in which the cell positions are not restricted to lattice positions (a discrete set of allowable points in the domain of computation). Furthermore, the domain of computation is arbitrary in this approach. The motion of the cells is partly deterministic as well as partly stochastic in the sense that cellular motion satisfies a stochastic differential equation (SDE). Cell death and cell division are also incorporated as stochastic processes. The model is suitable for simulating wound healing, as well as for invading colonies and tumor growth.

A drawback of the model (Vermolen and Gefen 2012) is the fact that the projection of the cells onto the substrate always stays circular. A repulsive force in order to prevent cells crawling on top of each other is incorporated on the basis of an invagination force from a model for viral infection of cells. The present paper will model cellular shape changes during cellular motion. In the present study, we only incorporate a chemical factor, but remark that mechanical pulling forces can be dealt with in an analogous manner. The combination of the two mechanisms (mechanical and chemical) will be dealt with in a future study. An interesting study (Neilson et al. 2011) models cell deformation as well as cell movement based on a pseudopod-based feedback. Their model consists of the solution of a system of partial differential equations on the cell surface by the use of a surface finite-element method. To displace the moving cell boundary, the level-set method is used in combination with a mechanical model. Their approach requires the solution of a coupled system of PDE's on the cell boundary, which evolves through the domain of computation, as well as a system of PDE's in the domain of computation. Their approach is rather computationally extensive, especially for a multi-cell case; however, their method is very elegant. Within this framework, we also mention the work by Barreira (2011), in which it is focused more more intensively on the surface finite-element method. This method is used to deal with Turing instabilities for pattern formation on evolving biological surfaces. Their approach is based on adaptive refinement of the surface finite-element mesh. Their approach can be used for various applications within developmental biology.

In this paper, we propose a relatively straightforward method to compute the evaluation of the cell shape during motion. First, we will give the mathematical framework, in which we first concentrate on one cell in the presence of a generic chemical source. Subsequently, we extend the model formulation to a multi-cell case in which the cells attract each other by the secretion and reception of a chemical signal, such as a growth factor. Subsequently, we describe the computational strategy for the solution of the mathematical problem. Then, we describe some of the numerical results, and we discuss the results using experimental data. Finally, we draw some conclusions.

\section{The mathematical model}

First, we formulate the analytic solution of a diffusion equation with a point source in an unbounded domain. Subsequently, we formulate the model for one cell, and finally, the case of a cell colony of a limited number of cells with attractors and receptors is dealt with.

\subsection{Solutions to diffusion equations for point sources}

Here, we consider solutions to diffusion equations with point sources. First, we treat the steady-state solutions, and subsequently, we will deal with transient solutions. The boundedness of the domain of computation can be incorporated by the use of (repeated) mirroring principles or by the use of Fourier Series Expansions upon avoiding finite-element discretizations.

\subsubsection{The quasi steady-state case}

For the release of the chemical agent that attracts cells, we assume all sources to be very small compared with cell areas, and therefore, we approximate these sources as point sources. 
Here, we will assume that a point source is able to move, but that the diffusion field that surrounds it sets in instantaneously. For this purpose, we consider a point source at $(X(t), Y(t))$, for which we have for $(x, y) \in \mathbb{R}^{2}$ and $t>0$

$$
-D \Delta c=\gamma(t) \delta(x-X(t), y-Y(t)),
$$

in which we assume that $(X(t), \mathbf{Y}(t))$ represents a certain determined trajectory, either following from a (biased) random motion or from an entirely deterministic motion. The concentration of the chemical is denoted by $c$, which diffuses with a diffusion coefficient $D$. Further, $\gamma$ denotes the strength of the point source, which may change in time, and $\delta($.$) denotes the Dirac Delta Function, which satisfies$

$$
\begin{aligned}
& \int_{\mathbb{R}^{2}} \delta(x, y) \mathrm{d} \Omega=1, \quad \text { with } \\
& \delta(x, y)=0, \quad \text { if }(x, y) \neq(0,0) .
\end{aligned}
$$

The solution to this differential equation is given by the following Green's Function

$c(t,(x, y))=-\frac{\gamma(t)}{2 \pi D} \ln \left((x-X(t))^{2}+(y-Y(t))^{2}\right)$,

in $\mathbb{R}^{2}$, which can be found in textbooks (Evans 1998). For the 3-D case, we report that the Green's Function is given by

$c(t,(x, y, z))=\frac{1}{4 \pi D\|\mathbf{x}-\mathbf{X}(t)\|}$,

where $\mathbf{x}$ and $\mathbf{X}(t)=(X(t), Y(t), Z(t))$, respectively, denote an arbitrary point and a trajectory of the point source in $\mathbb{R}^{3}$. We will elaborate a bit more on the two-dimensional version only. The 3-D elaboration goes analogously. In the case of multiple, say $n$, sources, with intensities $\gamma_{j}$ and positions $\left(X_{j}(t), Y_{j}(t)\right)$, linearity of the diffusion equation allows us to use the superposition principle, to obtain

$c(t,(x, y))=-\sum_{j=1}^{n} \frac{\gamma_{j}(t)}{2 \pi D} \ln \left(\left\|\mathbf{x}-\mathbf{X}_{j}(t)\right\|^{2}\right)$.

For a continuously distributed source-function $Q(t,(x, y))$ that is non-zero in $\Omega \subset \mathbb{R}^{2}$, we get the following convolution-based solution

$c(t,(x, y))=-\frac{1}{2 \pi D} \int_{\Omega} Q(t,(\bar{x}, \bar{y})) \ln \left(\|\mathbf{x}-\overline{\mathbf{x}}\|^{2}\right) \mathrm{d} \bar{\Omega}$,

where the above integral is evaluated over $(\bar{x}, \bar{y})$.

\subsubsection{The transient case}

For the release of the chemical agent that attracts cells, we proceed analogously to the steady-state case with the application of delta-functions to deal with the point sources, then we arrive at

$$
\begin{aligned}
& \frac{\partial c}{\partial t}-D \Delta c=\gamma(t) \delta(x-X(t), y-Y(t)), \\
& \text { for }(x, y) \in \mathbb{R}^{2}, \quad t>0,
\end{aligned}
$$

in which we assume that $(X(t), \mathbf{Y}(t))$ represents a certain determined trajectory, either following from a (biased) random motion or from an entirely deterministic motion. Further, $\gamma$ denotes the strength of the point source, which may change in time, and $\delta($.$) denotes the Dirac Delta Function$ again. Initially, the concentration is assumed to be zero, and then, the following solution for the 2-dimensional case, as a Green's Function, is derived according to the principles outlined in Evans (1998):

$c(t,(x, y))=\int_{0}^{t} \frac{\gamma(s)}{4 \pi D(t-s)} \exp \left(-\frac{\|\mathbf{x}-\mathbf{X}(s)\|^{2}}{4 D(t-s)}\right) \mathrm{d} s$,

for the two-dimensional case and

$$
\begin{array}{r}
c(t,(x, y, z))= \\
\int_{0}^{t} \frac{\gamma(s)}{(4 \pi D(t-s))^{3 / 2}} \exp \left(-\frac{\|\mathbf{x}-\mathbf{X}(s)\|^{2}}{4 D(t-s)}\right) \mathrm{d} s,
\end{array}
$$

for the 3-D case. Using this Green's Function, any solution with sources having a compact support, but non-zero measure can be constructed, or any initial condition, by the application of superposition arguments that result into a convolution. For completeness, we give the result for $n$ discrete point sources at the points $\left(X_{j}(t), Y_{j}(t)\right)$ and strength $\gamma_{j}(t)$ for $j \in\{1, \ldots, n\}$ :

$$
\begin{aligned}
c(t,(x, y))= & \sum_{j=1}^{n} \int_{0}^{t} \frac{\gamma_{j}(s)}{4 \pi D(t-s)} \\
& \times \exp \left(-\frac{\left\|\mathbf{x}-\mathbf{X}_{j}(s)\right\|^{2}}{4 D(t-s)}\right) \mathrm{d} s,
\end{aligned}
$$

as well as for a 'continuous' source that lives in $\Omega \subset \mathbb{R}^{2}$, we get the following solution by the use of convolution

$$
\begin{aligned}
c(t,(x, y))= & \int_{0}^{t} \int_{\Omega} \frac{Q(s,(\bar{x}, \bar{y}))}{4 \pi D(t-s)} \\
& \quad \times \exp \left(-\frac{\|\mathbf{x}-\overline{\mathbf{x}}\|^{2}}{4 D(t-s)}\right) \mathrm{d} \bar{\Omega} \mathrm{d} s .
\end{aligned}
$$

The above spatial integration is carried out over $(\bar{x}, \bar{y})$.

\subsection{The single-cell case}

We consider the projection of a cell with an initially specified geometry, size, and position. Inertia is neglected in the present formalism. The computational domain corresponds to a flat substrate, and hence, the domain is two-dimensional. The circumference of the projection of the cell is divided into 


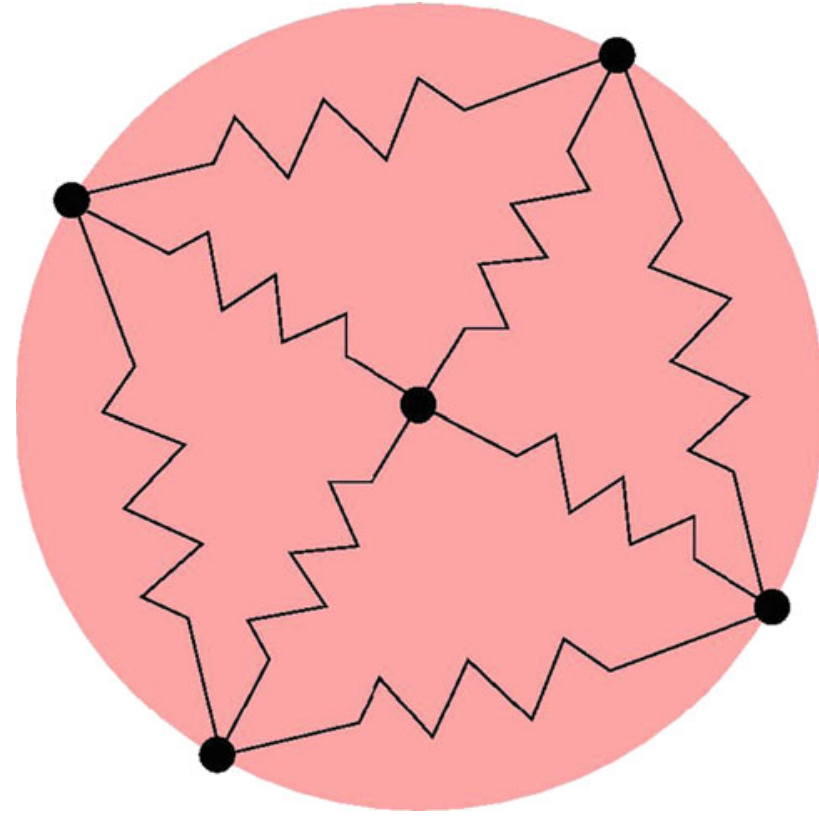

Fig. 1 A schematic of the distribution of springs that forms the backbone of the cell skeleton in the model

$N$ discrete points at which the cell senses the chemical signal and which is allowed to move according to the gradient of the chemical. As long as the distance between the cell boundary and the chemical source is sufficiently large in the sense that there is no physical contact, the velocity vector, $\mathbf{v}_{i}$, at point $i$ on the cell boundary is given by

$\mathbf{v}_{i}=\beta \nabla c\left(t, \mathbf{x}_{i}(t)\right), \quad$ for $i \in\{1, \ldots, N\}$.

Here, $\beta$ represents a mobility parameter of the cell boundary; this can be made spatially dependent over the cell boundary. At the present state of research, spatial variations over the cell boundary will not be incorporated. The concentration of the chemical agent that is emitted by the source is denoted by $c$. The gradient of the concentration is denoted by $\nabla c$. The position of the point $i$, which depends on time, is denoted by $\mathbf{x}_{i}(t)$. The velocity of the point is denoted by $\mathbf{v}_{i}$. The above equation guarantees that the velocity is directed toward the concentration gradient and that its magnitude depends on the magnitude of the concentration gradient. By this equation, one models chemotaxis, which is the cellular movement toward the gradient of a certain chemical.

Due to movement of the cell boundary toward the gradient of concentration of the chemical, the cell shape changes. Further, the area of the cell projection onto the substrate changes, even if one would assume that the cell volume stays constant. If the cell volume is assumed to be constant, then the three-dimensional cell shape would become flatter in the course of cellular motion. We assume that the cell boundary is connected to the cell center by the cytoskeleton, which is represented by a series of springs. Hence, we assume each boundary point to be connected to the cell center by a spring, see Fig. 1. The model is formulated such that the stable equilibrium shape of the projection of the cell in the absence of any biochemical stimulus, is a circle with radius $R$. Then, we get

$$
\begin{array}{r}
\mathbf{v}_{i}=\beta \nabla c\left(t, \mathbf{x}_{i}\right)+\alpha\left(\mathbf{x}_{c}(t)+\hat{\mathbf{x}}_{i}-\mathbf{x}_{i}(t)\right), \\
\text { for } i \in\{1, \ldots, N\},
\end{array}
$$

where $\alpha$ denotes the cell contraction forcing parameter. Further $\hat{\mathbf{x}}_{i}$ denotes the vector connecting the initial position of the points on the circumference of the projection of the cell (in 2-D), or on the surface of the cell (in 3-D), to the initial position of the center of the cell. This implies that the initial distances between neighboring points on the cell boundary is the (stable) equilibrium distance between these neighboring points. In other words, the initial distances between adjacent nodal points on the cell boundary will be recovered in the final stages and thereby the initial grid distribution is retained in the later stages. This provides a way of dealing with surface tension (hence tangential forces) since the positions of the meshpoints on the surface are determined by the distance to the cell center as well as by the initial distance between adjacent points. A key-assumption is that the surface energy and cell contraction is modeled by the same parameter. However, in the current approach, we do not need to solve a system of ODE's where the neighboring nodes participate, and hence, a PDE-like solution strategy is not needed. Furthermore, $\mathbf{x}_{c}(t)$ denotes the center of mass of the projection of the cell where the mass density is constant over the entire projection or surface in 3-D. Hence, this $\mathbf{x}_{c}(t)$ is given by

$\mathbf{x}_{c}(t):=\frac{\int_{\partial \Omega} \mathbf{x d} \Gamma}{\int_{\partial \Omega} \mathrm{d} \Gamma}$.

From the above Eqs. (13) and (14), it is clear that if $\beta=0$, then, $\mathbf{x}_{c}$ is constant and hence one obtains

$$
\lim _{t \rightarrow \infty} \mathbf{x}(t)=\mathbf{x}_{c}+\hat{\mathbf{x}}
$$

In the present formalism, we consider two cases: [1] where the cell does not consume the point source; and [2] where the cell does 'consume' the point source. The first case could be related to lamellipodia reaching a certain position in the extracellular environment. Further, the first case is considered because of the extension to the multi-cell case where we assume that the cells do not overlap. The second case is related to a cell engulfing a virus or bacterium, which is common for macrophages or phagocytes in any immune response. Another example could be an amoeba engulfing a smaller unicellular organism.

First, the cell is not allowed to 'consume' the point source, and therefore, we add a repulsive 'force' to avoid a condition where the cell comes too close to the source by adding the following repelling term 


$$
\begin{aligned}
\mathbf{v}_{i}= & \beta \nabla c\left(t, \mathbf{x}_{i}\right)+\alpha\left(\mathbf{x}_{c}(t)+\hat{\mathbf{x}}_{i}-\mathbf{x}_{i}(t)\right) \\
& +\frac{\varepsilon}{|\mathbf{x}-\mathbf{X}(t)|^{4}} \frac{\mathbf{x}-\mathbf{X}(t)}{|\mathbf{x}-\mathbf{X}(t)|}, \quad \text { for } i \in\{1, \ldots, N\}
\end{aligned}
$$

where $\mathbf{X}(t)$ represents the position of the point source, and $\epsilon$ is a constant that determines the range over which this repulsive force is active. Though this term is not the same as the repulsive force occurring in the Lennard-Jones potential for 'strong' nuclear forces, we were inspired by principles from nuclear physics for this term, see (Atkins and de Paula 2009) for a reference. It may as well model the repulsion exerted by the cellular membrane. In the case of multiple sources, we get

$$
\begin{aligned}
\mathbf{v}_{i}= & \beta \nabla c\left(t, \mathbf{x}_{i}\right)+\alpha\left(\mathbf{x}_{c}(t)+\hat{\mathbf{x}}_{i}-\mathbf{x}_{i}(t)\right) \\
& +\varepsilon \sum_{j=1}^{n} \frac{\mathbf{x}_{i}-\mathbf{X}_{j}(t)}{\left|\mathbf{x}_{i}-\mathbf{X}_{j}(t)\right|^{5}}, \quad \text { for } i \in\{1, \ldots, N\} .
\end{aligned}
$$

The repulsion term is also added for the multi-cell cases, where the cells are not allowed to physically overlap. We note that in the case where the cell is allowed to consume the point source, such as a bacterium, the term with $\varepsilon$ vanishes. Hence, this case is simply incorporated by setting $\epsilon=0$ and by subsequent removal of the point source from the simulation. To keep the area of the projection of the cell onto the two-dimensional substrate bounded, we assume that the cell-contraction force $\alpha$ depends on the cell-area by

$\alpha=\alpha_{0} \frac{\left(A_{0}-A_{\min }\right)\left(A_{\max }-A_{0}\right)}{\left(A(t)-A_{\min }\right)\left(A_{\max }-A(t)\right)}$,

which gives an increased driving force toward the equilibrium shape as the area $A$ of the projection of the cell differs more from the equilibrium area. Note that the above relationship is just phenomenological and hence not sustained by any experimental evidence. If experiments would indicate that this relation with $A_{\max }$ and $A_{\min }$ does not hold, then a modification can be made easily.

\subsection{The multi-cell case}

Next, we consider a set of $n$ cells, where each cell circumference is divided into $N$ grid points. Once again, inertia is neglected. The grid points on the $m$ th cell are denoted by $\mathbf{x}_{i}^{m}=\left(x_{i}^{m}, y_{i}^{m}\right)$ with corresponding velocity $\mathbf{v}_{i}^{m}$, for $i \in\{1, \ldots, N\}$ and $m \in\{1, \ldots, n\}$. All these $N$ points on a cell circumference act as a source of chemical that attract the grid points at the circumferences of all other cells. Since diffusion only takes place through the surface of the substrate, we assume that the diffusion field is not influenced by the presence of the cells. This is justified by the fact that the changes of the diffusivity due to traction is considered as a higher-order effect and hence the presence of the cells does not change the material characteristics of the underlying substrate. Note that the space between adjacent cells is assumed to be 'empty' or having passive transport properties, which does not contribute to the transport of the chemical signal. For the 3-D computations, the situation is slightly different. The cells are moving through the extracellular matrix, where their migration and deformations are dictated by the local concentration gradient of the attractant. In the present study, we use the same values for the diffusivities through the extracellular matrix and through the cells. This parameter setting allows the use of the 3-D Green's Functions. Since most of the cell body is composed of cytosolic fluid, the above assumption is reasonable, but in future modeling it can perhaps be assumed that the nucleus, which is a denser-likely more dense medium than the extracellular matrix in the case of soft tissues-is assigned different diffusivity properties. One additional factor that has not been considered in our simulations is the active transport mechanisms in cells, such as sodium-potassium pumps in the plasma membrane, which can potentially affect the intra-cellular diffusivity as well. Hence, the overall chemical signal that is sensed at a grid point of a certain cell is determined by the superposition of the chemical signals originating from the grid points at all the other cells (so not from the present cell).

The repulsive forces, from the grid points where the other cells are positioned, are the same as the ones described in the previous subsection. Further, the spring forces are dealt with analogously. We assume that if the area of the projection of the cell is smaller or larger than $A_{\min }$ and $A_{\text {max }}$, respectively, then this cell no longer emits the attracting chemical. In the present model, in which the spring /contraction force gets unbounded as the cell area approaches $A_{\min }$ or $A_{\max }$, this situation will never be reached in practice. Of course, large numerical time-steps would allow this state to happen. In the present work, we do not focus very much on any randomness of the motion of the grid points on the cell-edge. For completeness, we rephrase the equation for the velocity of each point, $i$, on each cell, $m$

$$
\begin{aligned}
& \mathbf{v}_{i}^{m}=\beta \nabla \tilde{c}^{m}\left(t, \mathbf{x}_{i}^{m}\right)+\alpha^{m}\left(\mathbf{x}_{c}^{m}(t)+\hat{\mathbf{x}}_{i}^{m}-\mathbf{x}_{i}^{m}(t)\right) \\
& +\varepsilon \sum_{l=1_{l \neq m}}^{n} \sum_{j=1}^{N} \frac{\mathbf{x}_{i}^{m}-\mathbf{x}_{j}^{l}(t)}{\left|\mathbf{x}_{i}^{m}-\mathbf{x}_{j}^{l}(t)\right|^{5}}, \\
& \text { for } i \in\{1, \ldots, N\}, \quad m \in\{1, \ldots, n\} \text {. }
\end{aligned}
$$

Here, $\tilde{c}$ refers to the concentration of attractor that is secreted by all cells except the current cell $m$; hence,

$$
\begin{aligned}
& \tilde{c}^{m}\left(t,\left(x_{i}, y_{i}\right)\right)=\frac{1}{4 \pi D} . \\
& \sum_{l=1_{l \neq m}}^{n} \sum_{j=1}^{N} \int_{0}^{t} \frac{\gamma_{j, l}(s)}{(t-s)} \exp \left(-\frac{\left|\mathbf{x}_{i}-\mathbf{x}_{j}^{l}(s)\right|^{2}}{4 D(t-s)}\right) \mathrm{d} s, \\
& \text { for } i \in\{1, \ldots, N\}, m \in\{1, \ldots, n\} .
\end{aligned}
$$


Here, $\gamma_{i, m}$ denotes the strength of the point source corresponding the $i$ th grid point on the $m$ th cell. The parameter $\gamma_{i, m}$ is normalized such that $\sum_{i=1}^{N} \gamma_{i, m}=\gamma_{0}$, for all $N$, in order to avoid dependence of the overall strength of each cell on the resolution in the limit as the number of nodes on the cell surface/circumference tends to infinity. A similar expression is derived for the steady-state case. In the present formalism, we note that if the cells would not secrete any chemo-attractant, then the cells would not have converged to each other. The repulsive forces, which the cells exert and which dominate once the inter-cellular distance becomes too small, prevent the cells from overlapping.

\subsection{Numerical solution method}

We explain the numerical method for the case of one cell in the presence of multiple point sources. At the end of this section, we present some 3-D simulations. To solve the resulting system of differential equations for both a single-cell and a multi-cell case, see Eq. (17), we divide the time into a discrete set of points, $t_{k}=k \Delta t$, where $\Delta t$ is the size of the time-step. Here, $k$ denotes the index indicating at which time the solution is computed. We use a rather standard procedure to solve the resulting set of ordinary differential equations, in which we combine the advantages of an implicit (backward Euler)- and explicit (forward Euler) method. First, we consider the quasi steady-state case. Then, for the gradient of the concentration, we get the following expression

$\nabla c(t,(x, y))=\frac{1}{2 \pi D} \sum_{j=1}^{n} \frac{\gamma_{j}(t)\left(\mathbf{X}_{j}(t)-\mathbf{x}\right)}{\left|\mathbf{X}_{j}(t)-\mathbf{x}\right|^{2}}$.

Considering Eq. (17), for $i \in\{1, \ldots, N\}$, this gives

$$
\begin{aligned}
\mathbf{x}_{i}^{k+1}= & \mathbf{x}_{i}^{k+1}+\Delta t\left(\beta \nabla \hat{c}_{i}^{k+1}+\alpha\left(A^{k}\right)\right. \\
& \left.\times\left(\mathbf{x}_{c}^{k}(t)+\hat{\mathbf{x}}_{i}-\mathbf{x}_{i}^{k+1}(t)\right)\right) \\
& +\Delta t \varepsilon \sum_{j=1}^{n}\left(\frac{\mathbf{x}_{i}^{k+1}-\mathbf{X}_{j}\left(t^{k+1}\right)}{\left|\mathbf{x}_{i}^{k}-\mathbf{X}_{j}\left(t^{k+1}\right)\right|^{5}}\right),
\end{aligned}
$$

for the determination of the solution at the next time-step $k+1$ using the values at the previous time-step $k$. Note that the subscript $k$ denotes the time-step, and hence, the notation differs from the previous subsection. Furthermore, $\nabla \hat{c}^{k+1}$ is defined by

$\nabla \hat{c}^{k+1}=\frac{1}{2 \pi D} \sum_{j=1}^{n} \frac{\gamma_{j}\left(t^{k+1}\right)\left(\mathbf{X}_{j}\left(t^{k+1}\right)-\mathbf{x}^{k+1}\right)}{\left|\mathbf{x}^{k}-\mathbf{X}_{j}\left(t^{k+1}\right)\right|^{2}}$.

From this equation, a linear equation for $\mathbf{x}_{i}^{k+1}$ is solved at each time-step, which makes the method implicit, and more stable than a classical explicit method where all occurrences of $\mathbf{x}_{i}$ are computed at the previous time-step $k$. In the case of cells approaching each other such that the repulsive forces get more important, it is necessary to decrease the time-step such that the maximum distance that is travelled by a grid point is smaller than $\varepsilon$. Hence, to this extent an adaptive time-step has been implemented into the simulations.

For the transient case, we need to integrate the gradientterm over time, which gives

$$
\begin{aligned}
\nabla c(t,(x, y))= & \sum_{j=1}^{n} \int_{0}^{t} \frac{\gamma_{j}(s)\left(\mathbf{x}-\mathbf{X}_{j}(s)\right)}{8 \pi D^{2}(t-s)^{2}} \\
& \times \exp \left(-\frac{\left|\mathbf{x}-\mathbf{X}_{j}(s)\right|^{2}}{4 D(t-s)}\right) \mathrm{d} s,
\end{aligned}
$$

In Eq. (22), we insert for $\nabla \hat{c}^{k+1}$ :

$$
\begin{aligned}
\nabla \hat{c}_{i}^{k+1}= & \frac{1}{8 \pi D^{2}} \cdot \\
& \sum_{j=1}^{n} \int_{0}^{t^{k+1}} \frac{\gamma_{j}(s)\left(\mathbf{x}_{i}^{k+1}-\mathbf{X}_{j}(s)\right)}{\left(t^{k+1}-s\right)^{2}} . \\
& \times \exp \left(-\frac{\left|\mathbf{x}_{i}^{k}-\mathbf{X}_{j}(s)\right|^{2}}{4 D\left(t^{k+1}-s\right)}\right) \mathrm{d} s .
\end{aligned}
$$

Again, from this equation, a linear equation for $\mathbf{x}_{i}^{k+1}$ is solved at each time-step, which makes the method implicit, and more stable than a classical explicit method where all occurrences of $\mathbf{x}_{i}$ are computed at the previous time-step $k$. In the case of cells approaching each other such that the repulsive forces get more important, it is necessary to decrease the time-step such that the maximum distance that is travelled by a grid point is smaller than $\varepsilon$. Hence, to this extent, an adaptive time-step has been implemented into the simulations.

For the 3-D simulations, we divide the original spherical surface into a set of $n$ discrete points using a surface mesh generator (Segal 2012)). The neighboring discrete points are arranged into a triangular surface mesh, of which the topology, that is, the arrangement of the vertices and the $n_{\mathrm{el}}$ triangular elements, is stored in memory. This storage enables us to evaluate the integrals that are necessary to compute the center of mass, cell area and volume, see Eq. 14. For the sake of illustration, we elaborate on the computation of the numerator in the expression for the coordinates of the cell center,

$$
\int_{\partial \Omega} \mathbf{x} \mathrm{d} \Gamma \approx \sum_{k=1}^{n_{\mathrm{el}}} \int_{\partial \Omega_{k}} \mathbf{x} \mathrm{d} \Gamma
$$

Each integral over element $\partial \Omega_{k}$ in the above equation is evaluated using Newton-Cotes numerical quadrature. This is also carried out for the post-processed quantities like the cell volume and surface area that are needed to obtain the Cell Shape Index (see Sect. 3). An example of a meshed surface of the cell is shown in Fig. 2. 


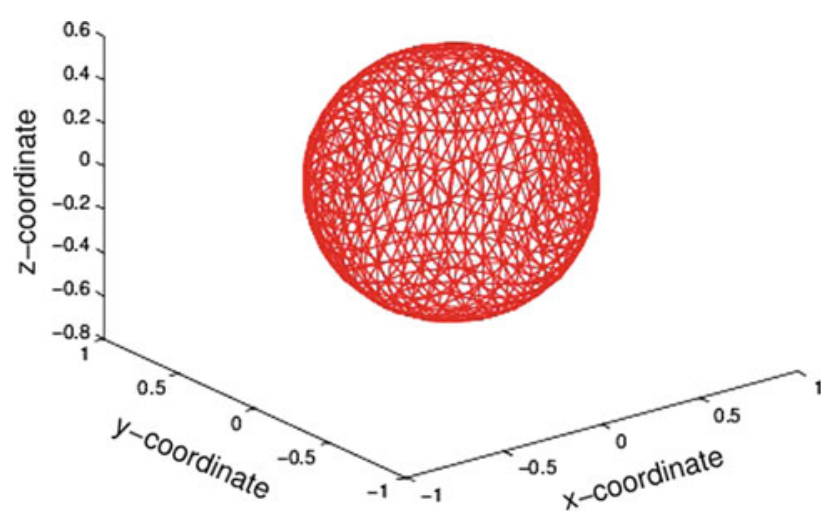

Fig. 2 The division of nodes on the spherical ground configuration of the cell. The number of nodes and elements is 588 and 50, respectively

Table 1 Values for the various parameters used in the planar simulations

\begin{tabular}{lll}
\hline Parameter & Value & Unit \\
\hline$\beta$ & 1 & $\mathrm{~mm}^{4} / \mathrm{h} / \mathrm{mol}$ \\
$\gamma$ & 1 & $\mathrm{~mol} / \mathrm{mm}^{3} / \mathrm{h}$ \\
$\varepsilon$ & 0.01 & $\mathrm{~mm}^{6} / \mathrm{h}$ \\
$D$ & $\frac{1}{2 \pi}$ & $\mathrm{mm}^{2} / \mathrm{h}$ \\
$\alpha_{0}$ & 1 & $1 / \mathrm{h}$ \\
$A_{\min }$ & $\frac{A_{0}}{\sqrt{2}}$ & $\mathrm{~mm}^{2}$ \\
$A_{\max }$ & $A_{0} \sqrt{2}$ & $\mathrm{~mm}^{2} / \mathrm{s}$ \\
\hline
\end{tabular}

\section{Computer simulations}

In the present computer simulations, we use the quasi steadystate solutions to the diffusion equations, unless stated otherwise. The quasi steady-state is used since it does not require the temporal integration, which makes the calculations faster. First, we evaluate the model using one cell and several external point sources, for which motion is deterministic or random. Subsequently, we consider a case of multiple cells. In all the computational results, we use the input-data from Table 1.

As numerical settings, we typically used 20 points over each cell circumference, and a time-step of $0.025 \mathrm{~h}$. Doubling of the number of points and halving the time-step hardly influenced the simulations, so numerical convergence was reached. Times were in hours and spatial dimensions are in micron. For the interpretation of the results, we consider both the area of the cell and the Cell Shape Index (CSI). For a given region $\Omega \subset \mathbb{R}^{2}$, with boundary $\partial \Omega$, the CSI is defined as

$\operatorname{CSI}(\Omega)=\frac{4 \pi A(\Omega)}{l^{2}(\partial \Omega)}$,

in the two-dimensional case. Further, for the 3-D case, we arrive at

$\operatorname{CSI}(\Omega)=\frac{6 \sqrt{\pi} V(\Omega)}{(A(\partial \Omega))^{3 / 2}}$.
Using the Divergence Theorem, the area of $\Omega$, denoted by $A(\Omega)$ is computed by

$A(\Omega)=\int_{\partial \Omega} x n_{x} \mathrm{~d} \Gamma$,

where $\mathbf{n}=\left\langle n_{x}, n_{y}\right\rangle$ represents the outward pointing unit normal vector on $\partial \Omega$ in two dimensions. In three dimensions, a similar expression is used. We use the second expression for reasons of numerical accuracy. Furthermore, $l(\partial \Omega)$ denotes the length of the boundary curve $\partial \Omega$. Note that a circle gives a CSI of one, and a line gives a CSI of zero.

\subsection{A single-cell case}

We consider a cell with initial, equilibrium projected area of $0.2 \mu \mathrm{mm}^{2}$, hence $R=\sqrt{\frac{0.2}{\pi}}$. Note that this cell size is arbitrary and that all the calculations can be scaled to represent any single-cell micro-organism, from very small prokaryotes (e.g., bacteria with radii of around 0.1 micron) up to animal or human eukaryotic cells (order of 10's-of-microns) or even protozoa, for example, amoeba (order of 100's-of-microns). In the present paper, we just aim at giving a phenomenological description. Further, the initial center of mass is located at $(x, y)=(0,0)$. In the first simulation, we show the evolution of the shape of the projection of the cell under the influence of four point sources. These point sources travel according to the following parametric equations:

$X_{1}(t)=-1.5+0.95 \sin (2 \pi t)$,

$Y_{1}(t)=0$,

$X_{2}(t)=1.5-0.95 \sin (2 \pi t)$,

$Y_{2}(t)=0$,

$X_{3}(t)=0$,

$Y_{3}(t)=-1.5+0.95 \sin (2 \pi t)$,

$X_{4}(t)=0$,

$Y_{4}(t)=1.5-0.95 \sin (2 \pi t)$.

The behavior of the cell shape and the migration of the cell is shown in Fig. 3 at consecutive times. In the beginning, the point sources have no significant influence on the cell, and then, the cell boundary does not move. In the course of time, the sources approach the cell boundary by which the cell boundary starts 'feeling' the chemical that is emitted by the point sources. As the point sources move away from the cell, see after 390 time-steps for instance, then the cell boundary retreats back to normal configuration. This can also be seen in Fig. 4 for a larger time-frame. It can be seen clearly that the elongation of the cell makes the area increase and CSI decrease, subsequently when the point sources vanish that the cell area and CSI decrease back to their normal equilibrium 

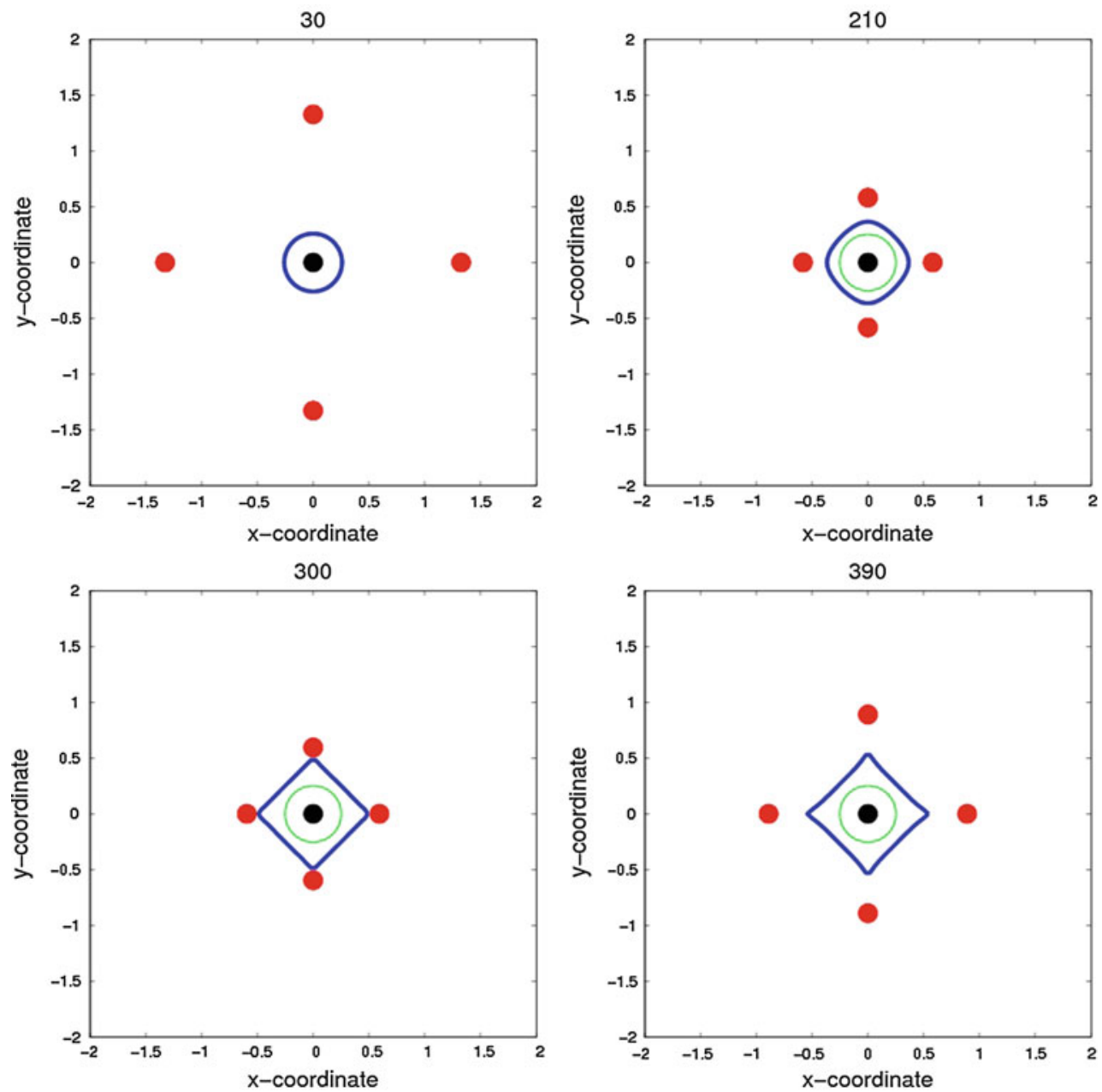

Fig. 3 The blue line represents the shape of the projection of a cell under the influence of four traveling point sources after 30, 210, 300, and 390 time-steps for four point sources. The red dots are the locations

of the point sources, the black dot in the center corresponds to the center of mass of the cell. The green line depicts the initial position of the projection of the cell boundary
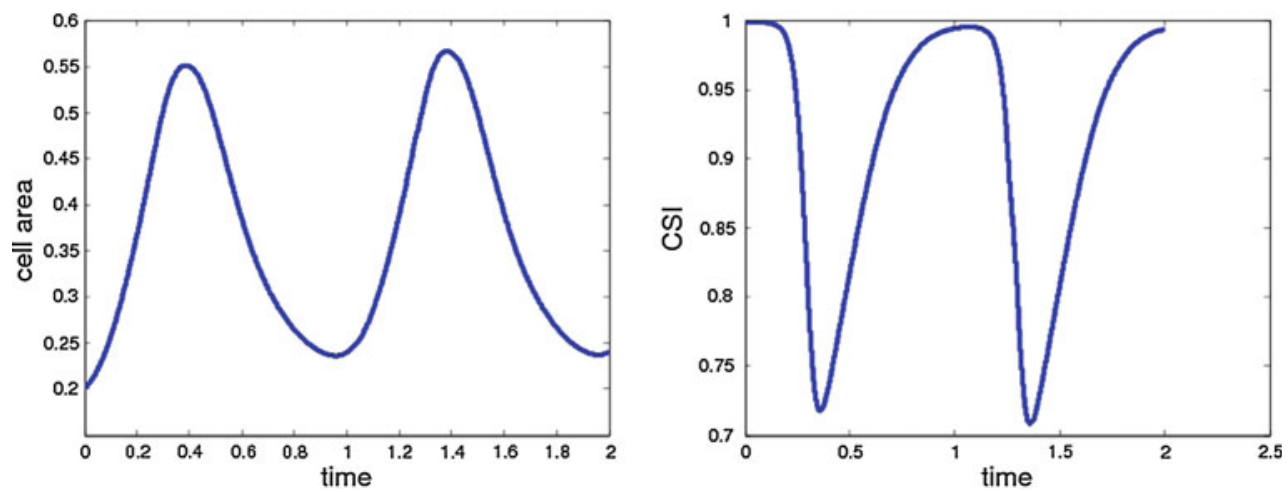

Fig. 4 The area (left) and CSI (right) of the cell as a function of time under the presence of four oscillating point sources

values. The decrease of the CSI during cell motion is also observed experimentally (Lin and Helmke 2009).

In the second run, we consider one chemical point source, whose position is parametrized by

$X_{1}(t)=-1.5+0.55 \sin (2 \pi t), \quad Y_{1}(t)=0$.
In Fig. 5, it can be seen that the cell deforms and moves toward the chemical point source. In this model, the chemical point source is treated as a solid sphere whose movement is not disturbed by the cell. On the contrary, the cell boundary is pushed away if the chemical point source is too close to 

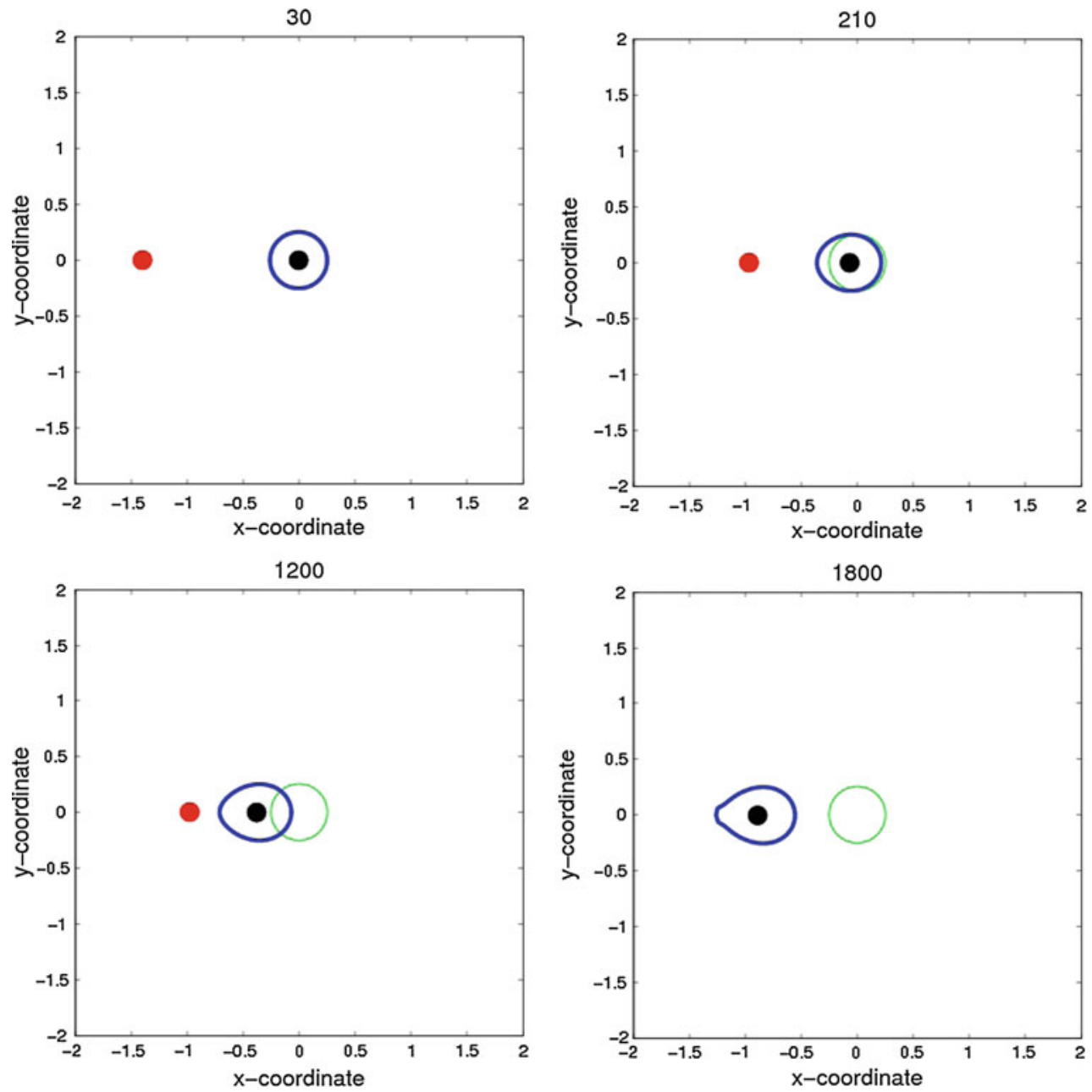

Fig. 5 The blue line represents the shape of the projection of a cell under the influence of one traveling point source after 30,210, 1,200, and 1,800 time-steps for one point source. The red dot is the locations

of the point source, the black dot in the center corresponds to the center of mass of the cell. The green line depicts the initial position of the projection of the cell boundary
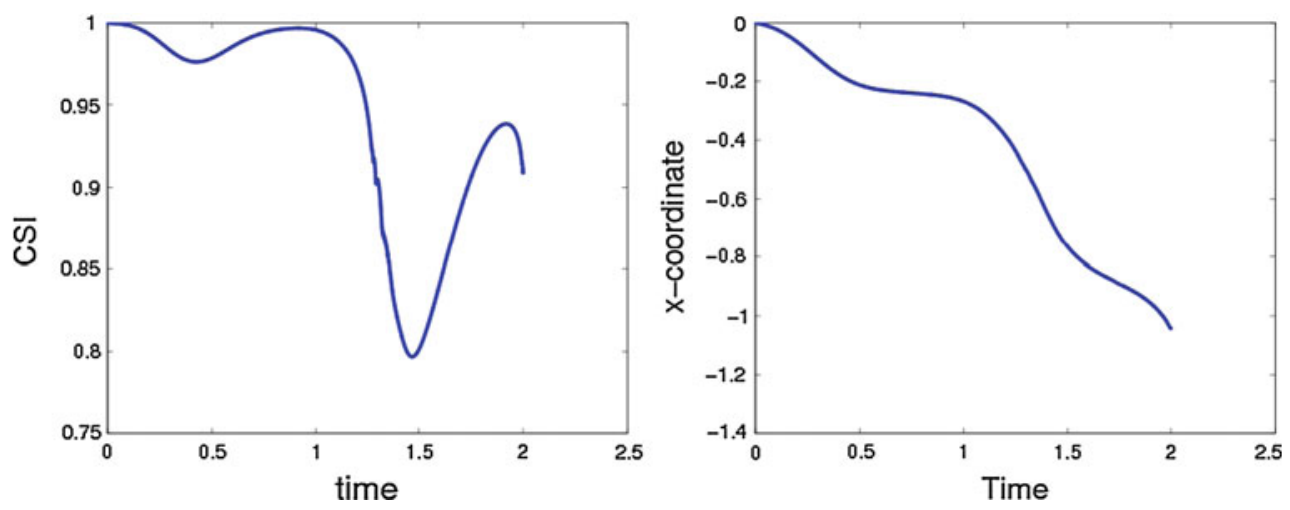

Fig. 6 The CSI of the cell as a function of time under the presence of one alternatingly moving point source, and the $x$-coordinate of the center of mass as a function of time

the cell boundary. In other words, hard impingement occurs. This explains the non-sharp 'nose' of the cell at time 1800, see Fig. 5. In Fig. 6, we plot the CSI and the $x$-coordinate of the cell center as a function of time. It can be seen that the cell area increases due to the deformation and translation of the cell resulting from the non-symmetric nature of the point 

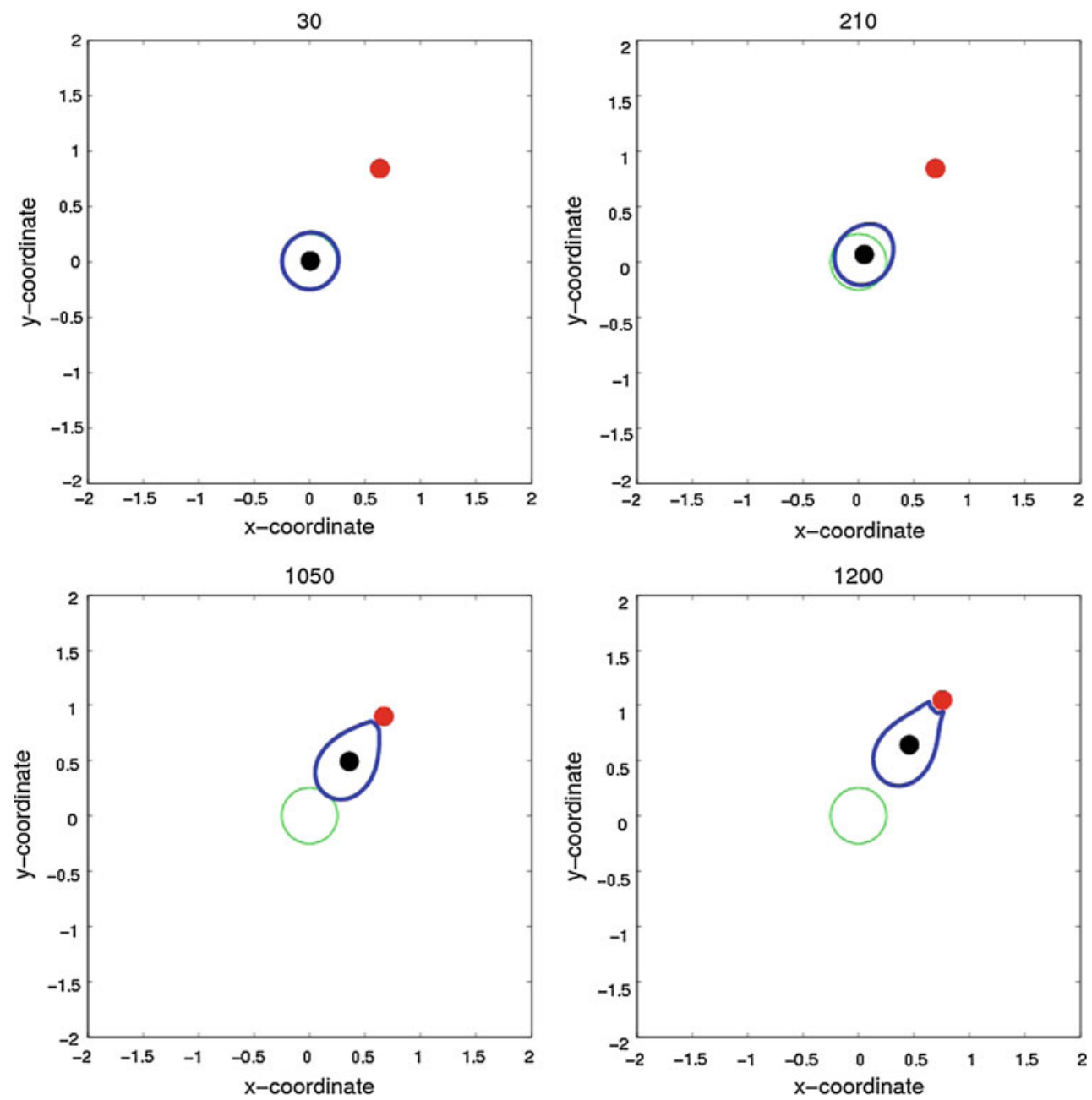

Fig. 7 The blue line represents the shape of the projection of a cell under the influence of one randomly moving point source after 30, 210, 1,050 , and 1,200 time-steps for a randomly moving chemical point

source. The red dot is the locations of the point source, the black dot in the center corresponds to the center of mass of the cell. The green line depicts the initial position of the projection of the cell boundary

source. Further, it can be seen that the cell moves to the left in an oscillatory manner. In the last run of simulations, we consider the random motion of one point source, given by

$\mathrm{d} X_{1}(t)=\mu \mathrm{d} W(t), \quad \mathrm{d} Y_{1}(t)=\mu \mathrm{d} W(t)$

where $\mathrm{d} W(t)$ denotes a stochastic parameter (Wiener Process) with range in $[-1,1]$ and $\mu$ denotes the maximum velocity of the chemical point source. The results are shown in Fig. 7. The chemical attracts the cell, by which the cell is deformed. Subsequently, we assume that the cell cannot 'consume' the source and the cell and the source are repelled from each other if the distance is too close. In this example, the motion of the point source is directed away from the cell if the distance between the point source and cell boundary is too small. As the source has moved away from the cell, the source is allowed to move randomly. Due to approaching of the cell to the source, the cell 'pushes' the chemical point

source away. This also shapes the curved structure of the cell tip near the point source at the latest stages.

\subsection{A multiple cell case}

Next, we consider the case of multiple cells. In the current simulation, we use seven cells, of which the centers are located at the following points:

$$
\begin{aligned}
& \left(x_{1}, y_{1}\right)=(0,-1.3), \quad\left(x_{2}, y_{2}\right)=(1,0), \\
& \left(x_{3}, y_{3}\right)=(0,1.67), \quad\left(x_{4}, y_{4}\right)=(-1.25,-0.2) \\
& \left(x_{5}, y_{5}\right)=(-0.1,0.05), \quad\left(x_{6}, y_{6}\right)=(-0.75,0.75), \\
& \left(x_{7}, y_{7}\right)=(0.3,0.7) .
\end{aligned}
$$

We show some snapshots at various times in Fig. 8. Note that the initial positions and shapes are displayed as thin green lines in the figure. First, the cells converge to each other due to chemical attraction. At a later stage, they are influenced 

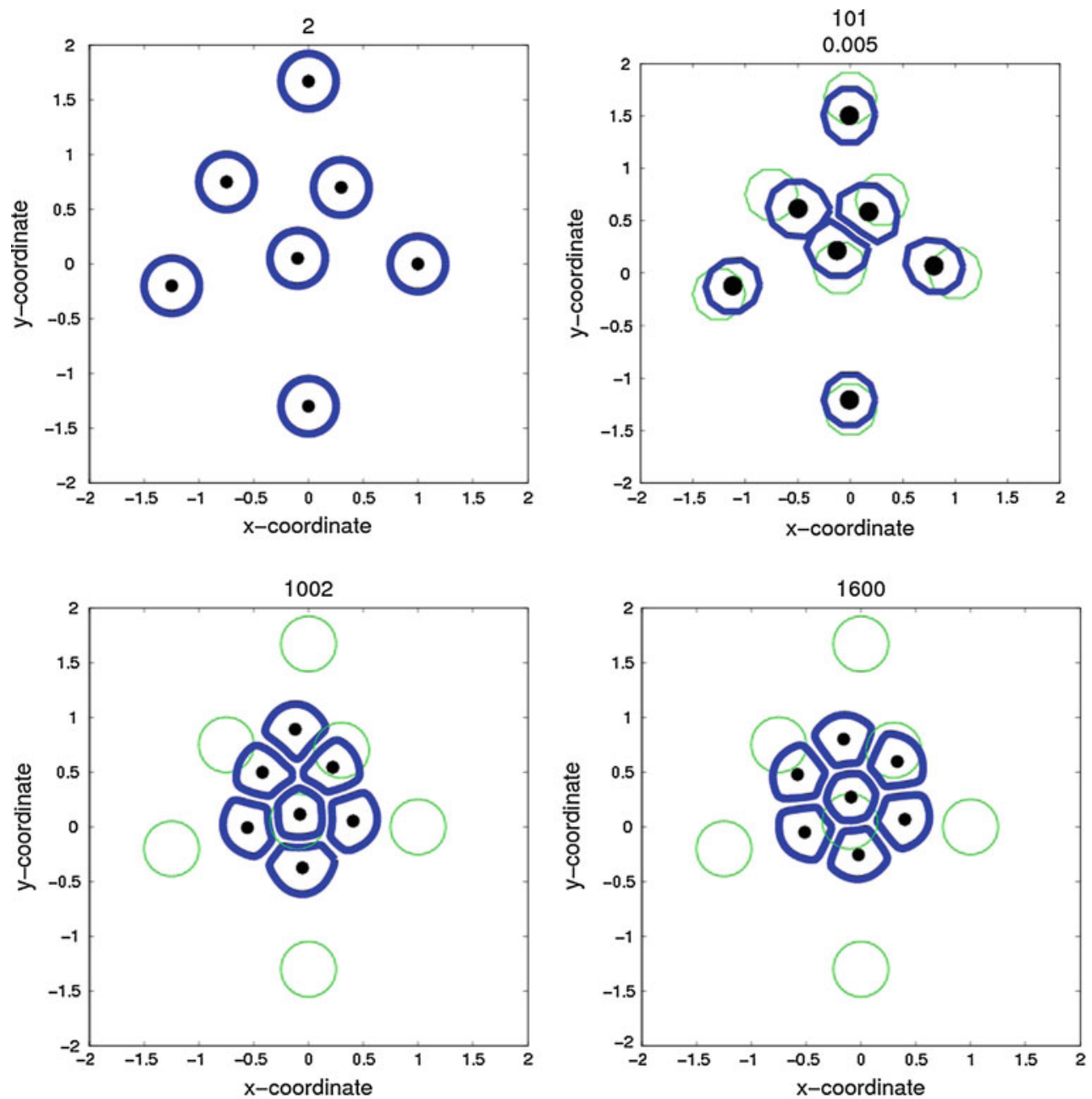

Fig. 8 A multi-cell configuration with initial positions as in Eq. (33). Here snapshots are shown at 0, 100, 1,000 and 1,600 time-steps. The time-step was taken at 0.025 . The colony gradually becomes spherical with predominantly non-circular border cells

by impingement and then the repulsive forces become dominant. At the latest stage, the cells are packed together and the overall shape of the collection of cells converges to a circular shape, although the individual cells are not circular. This will be the final equilibrium shape. The numerical calculations suggest that this equilibrium configuration is stable with respect to perturbations. To confirm this behavior, we show another run with different initial positions that are almost located on a straight line. The initial positions are given by

$$
\begin{aligned}
& \left(x_{1}, y_{1}\right)=(-1.5,0), \quad\left(x_{2}, y_{2}\right)=(-1,0), \\
& \left(x_{3}, y_{3}\right)=(-0.5,0), \quad\left(x_{4}, y_{4}\right)=(0,0.2) ; \\
& \left(x_{5}, y_{5}\right)=(0.5,0), \quad\left(x_{6}, y_{6}\right)=(1,0), \\
& \left(x_{7}, y_{7}\right)=(1.5,0) .
\end{aligned}
$$

This configuration, although the initial state is such that almost all cells are located on a straight line, gives a similar end-state as in the previous case. The evolution of the cell positions and shapes is shown in Fig. 9. Here, it can be seen that the state converges to a circular colony once again, in which all the border cells have the same non-circular geometry. The central cell is hexagonal, like in the previous example. The polygonal shapes that we observe in the simulations are in qualitative agreement with the experimental observations (Axelrod 2006). We show the evolution of the CSI of the initially most-left positioned cell and of the central cell in Fig. 10. The CSI of all the cells except the central tends to a similar value as $t \rightarrow \infty$. It can be seen that as time proceeds, the central cell will mostly resemble a circular shape, which is consistent to the CSI of a regular octagon, which has a CSI of approximately 0.95 . The decrease of the CSI is in line with the observations by (Gray et al. 2002). Finally, we show some preliminary results for a small cell colony with 100 cells. Here, the same data as in Table 1 were used and the initial cell radius is 0.4 . On each cells 20 gridnodes were positioned. The cells were somewhat smaller than in the previous calculations. The CPU time was approximately $30 \mathrm{~min}$ for 175 time-steps of magnitude 0.01 with 125 cells. 

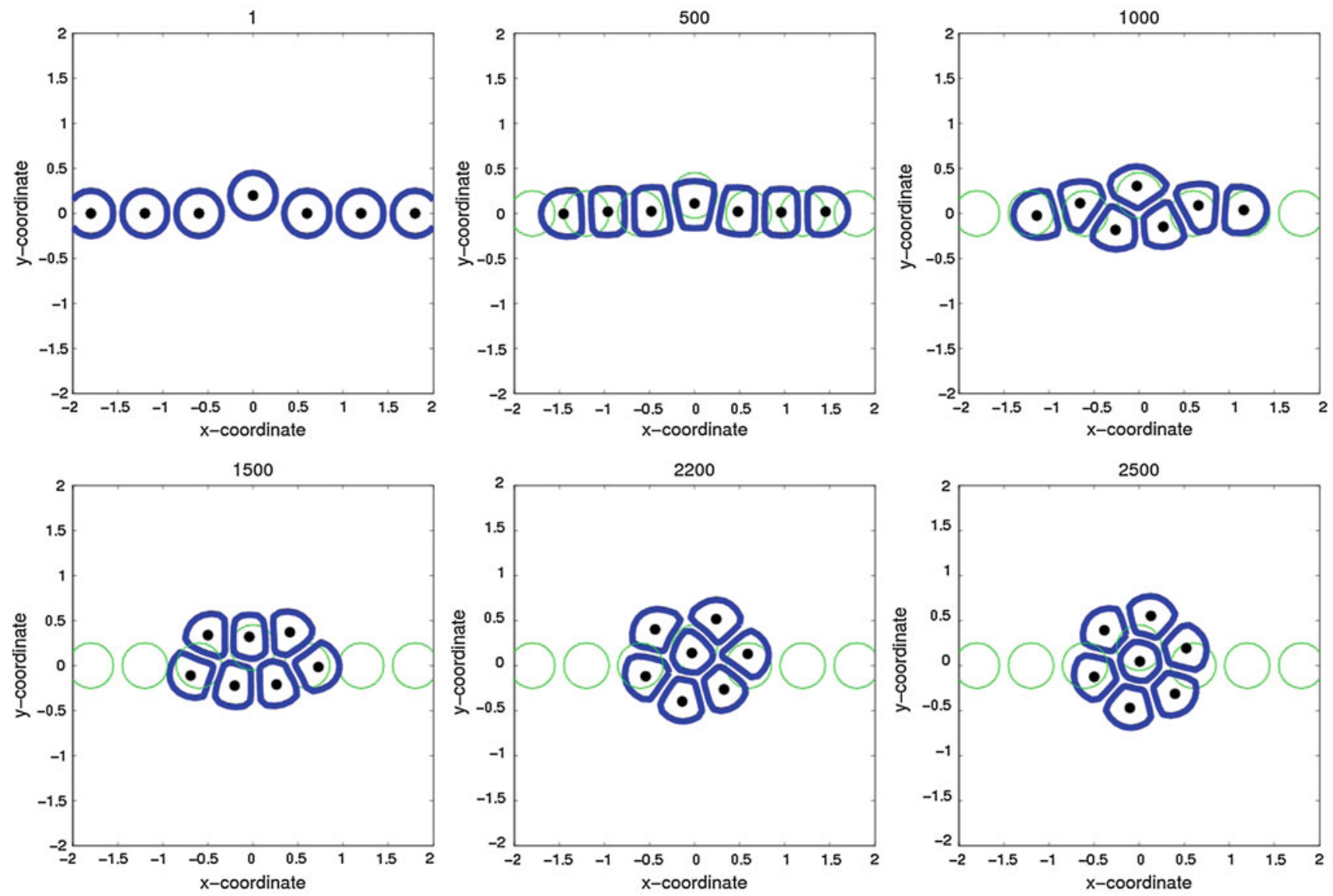

Fig. 9 A multi-cell configuration with initial positions as in Eq. (34). Here snapshots are shown at 0, 500, 1,000, 1,500, 2,200 and 2,500 time-steps. The time-step was taken at 0.025 . The colony gradually becomes spherical with predominantly non-circular border cells

The results are shown in Fig. 11. It can be seen that the cells obtain cobblestone shapes and that the colony stays about circular of shape. Upon introducing small perturbations of the initial cell positions, the final state of the simulations hardly changes.

It is interesting to investigate this issue further in the future and to see whether the predicted behavior agrees with biological observations.

\subsection{A repulsion case}

It is well-known that some chemicals do not attract cells, but merely repel them. In Fig. 12, we show the evolution of a repelled cell at various stages. Here the source that secretes the chemical moves in a random fashion. It can be seen that the boundary of the cell that is closest to the chemical source, moves away at the highest speed. The cell area stays reasonable due to the constraint that the binding force of a cell increases nonlinearly if the cell gets too much compressed. As the cell moves away from the source, the geometry becomes more circular.

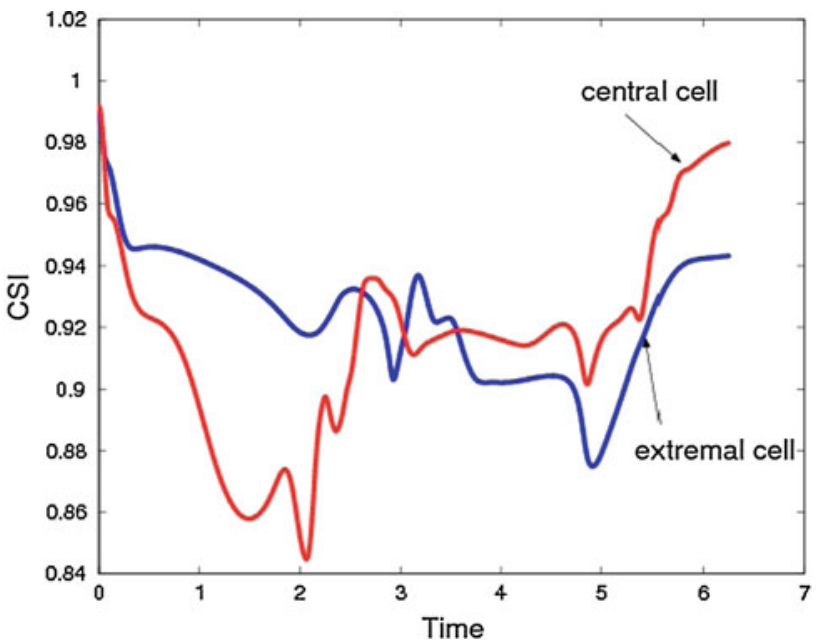

Fig. 10 The CSI of the central cell (red) and of the cells that initially resided on the very left (blue)

\subsection{Three-dimensional simulations}

In this section, we consider an initially spherical cell that is exposed to deformation and migration as a result of a 


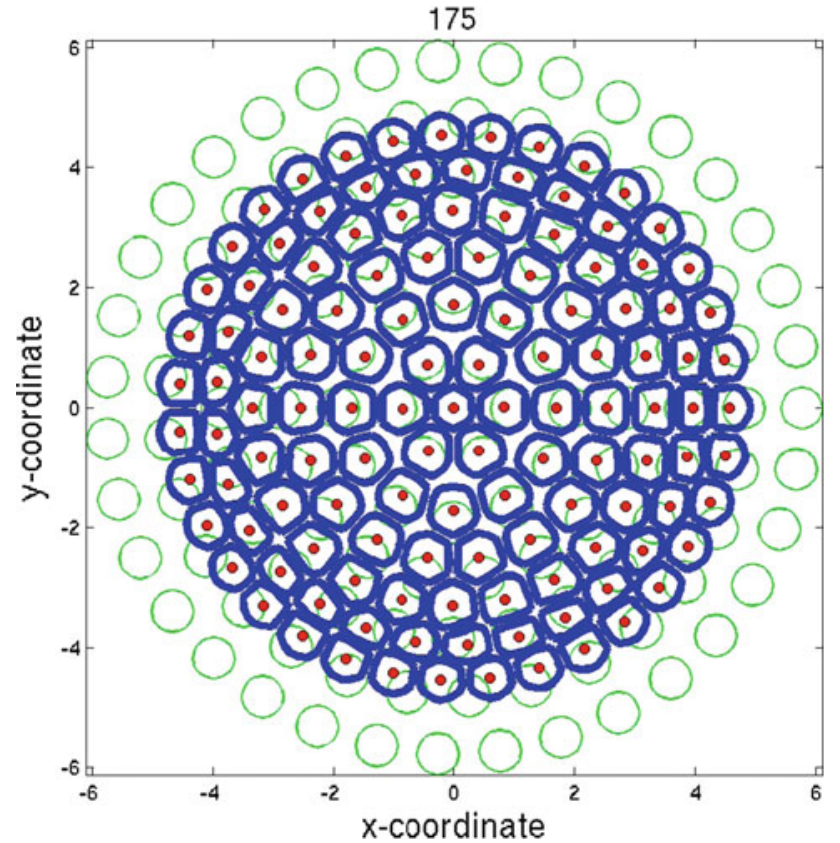

Fig. 11 The rearrangement of a colony of 125 cells at time-step 175 . The time-step is 0.01 . The thick (blue) lines represent the cells at the final state of the simulation. The thin (green) lines represent the initial positions of the cells

Table 2 Values for the various parameters used in the three-dimensional simulations

\begin{tabular}{lll}
\hline Parameter & Value & Unit \\
\hline$\beta$ & 5 & $\mathrm{~mm}^{4} / \mathrm{h} / \mathrm{mol}$ \\
$\gamma$ & 1 & $\mathrm{~mol} / \mathrm{mm}^{3} / \mathrm{h}$ \\
$\varepsilon$ & 0.01 & $\mathrm{~mm}^{6} / \mathrm{h}$ \\
$D$ & $\frac{1}{4 \pi}$ & $\mathrm{mm}^{2} / \mathrm{h}$ \\
$\alpha_{0}$ & 1.5 & $1 / \mathrm{h}$ \\
\hline
\end{tabular}

chemical stimulus due to the release of an attractant from a bacterium. To highlight the performance of the model, we used the data from Table 2.

For the sake of simplicity, we use a constant cell contraction force parameter $\alpha$. In the present simulations, we assume that once the cell contacts the bacterium, then the bacterium is consumed or neutralized and hence it no longer exerts any attraction to the cell. We show some snapshots at several times for various cases. The first case is plotted in Fig. 13, where the interaction with one fixed bacterium is considered. It can be seen that first the cell deforms and moves toward the bacterium according to the local concentration gradient. Subsequently, the bacterium is engulfed and the cell shape gradually changes back to the equilibrium spherical configuration. In the meanwhile, the cell has moved toward the bacterial source, and once the bacterium has been neutralized, the center of mass no longer moves.
The second case is plotted in Fig. 14, where a cell is exposed to six bacterial sources. It can be seen that first, the cell deforms in a starfish manner in order to be able to reach the bacteria and to consume them at the same time. At the final stages, the cell shape evolves back to the equilibrium spherical geometry. In this run, the cell center did not move.

The third case is plotted in Fig. 15, where a cell is exposed to three bacterial sources. Due to asymmetry, the cell center first moves in the $y$-direction. Further, the cell deforms in order to reach the bacteria. After consumption of the bacteria, the cell shape evolves back to the equilibrium spherical configuration, where the cell center has moved a bit in the $y$-direction.

The results of the last 3-D simulation are shown in Fig. 16, where once again three bacterial sources are modeled. However, now the cell stiffness has increased by a factor 100, by which the cell is barely able to deform. Only if the cell gets in very close contact with the bacteria, the cell shape changes in order to reach the bacteria. The reason for this rapid shape change is that the concentration gradient from the Green's Function gets very large. Since the cell hardly deforms, the cell reaches the bacteria by means of migration. Hence, in this simulation, the migration of the cell is more significant than in the previous case, which is shown in Fig. 15. Now the cell is moving to all the bacterial sources, as can be observed in Fig. 16.

Finally, we plot the CSI for the 3-D cell versus time in Fig. 17. For the cases of a low cell stiffness, that is, a low $\alpha$-value, in both cases with three and six bacterial sources, it can be seen that the pattern is similar since cell deformation is the mechanism to engulf the bacteria. However, if the stiffness of the cell increases, then the cell has to move more and to deform less in order to consume the bacteria. This is seen in Fig. 17 as well. In the last case, the changes in the CSI are therefore less significant.

\section{Discussion and relevant experimental observations}

The model that we showed so far describes the evolution of the cell shape and position in a realistic way. The concentration gradient is assumed to be the determining factor for cell motion, and therefore the transient version of the Green's Functions may give slightly more realistic results if the point sources move 'fast' compared to the diffusion rate. In our case, we used a high value for $D$, which means that diffusion is much faster than the movement of the sources. The temporal changes of the concentration are hence determined by the movement of the sources and not so much by diffusion itself. However, if $D$ is low, then, the transient model should be considered. In this case, the motion of the cells may not be directed toward the source, since the concentration gradient does not need to be parallel to 

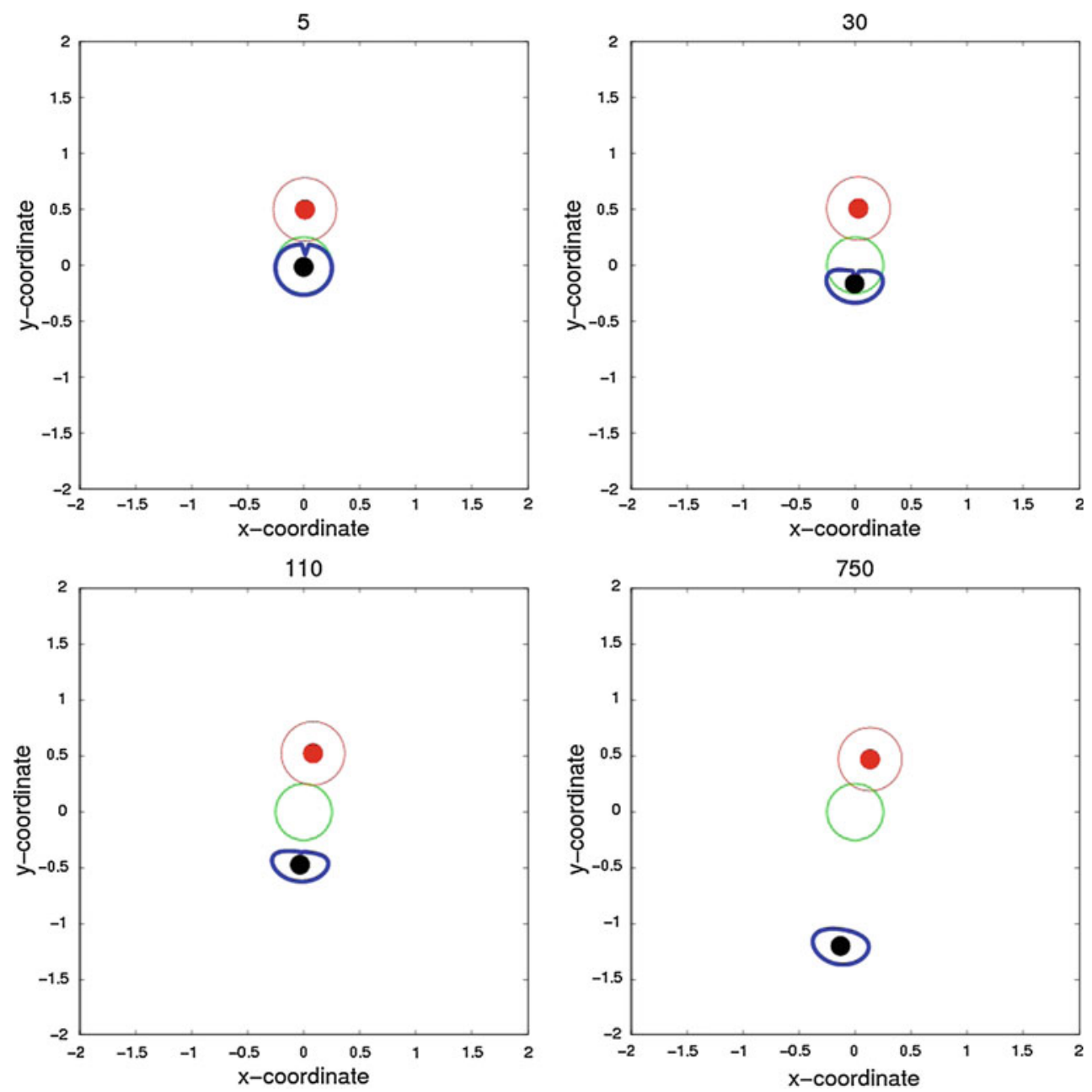

Fig. 12 A repelled cell from a chemical at various stages, at 5, 30, 110 and 750 time-steps

the line connecting the point at the cell boundary and the point source. The extension to a transient diffusion field is relatively straightforward, though it will increase the parameter space a bit and barely change the model implications as such. Further, in the multi-cell case, it will need a timeintegration operation where the entire history of the positions of all points at the cell surface is needed. Therefore, this issue is not addressed in the multi-cell case in the present study. However, for the sake of illustration, we show some snapshots of a 3-D cell at various times where the transient Green's Functions are used with one moving bacterium. The equation of motion for the bacterium is given by

$$
\begin{aligned}
& X(t)=-1.75 \\
& Y(t)=1.5 \sin (4 \pi t), \\
& Z(t)=0 .
\end{aligned}
$$

In this particular example, we have chosen a high migration speed for the bacterium. By this effect, we see that the concentration first has to build up so that the cell only starts moving at a relatively late time compared with the non-transient case. Further, we see that the cell deformation kinetics exhibit a memory behavior since the motion is not necessarily directed toward the bacterium, but to an earlier position of the bacterium, see Fig. 18.

Furthermore, with some extensions, we believe that it is possible to include alternative (from circular) equilibrium shapes of the cells, corresponding to different phenotypes. We also expect that it is possible to extend the model such that the cell geometry exhibits alternative shapes from oval geometries using a point-dependent sensitivity of the philapods. This could be done by solving Turing or Cahn-Hilliard instability problems using moving ALE-based surface finiteelement methods, like it has been done (Neilson et al. 2011). These Turing/Cahn-Hilliard instability problems could be solved for the gridnodes on the cellular boundary, where a reaction-term follows from the chemical signal that is emitted by the outer sources such as bacteria or other cells. The pattern that is obtained from these equations can be used 
1

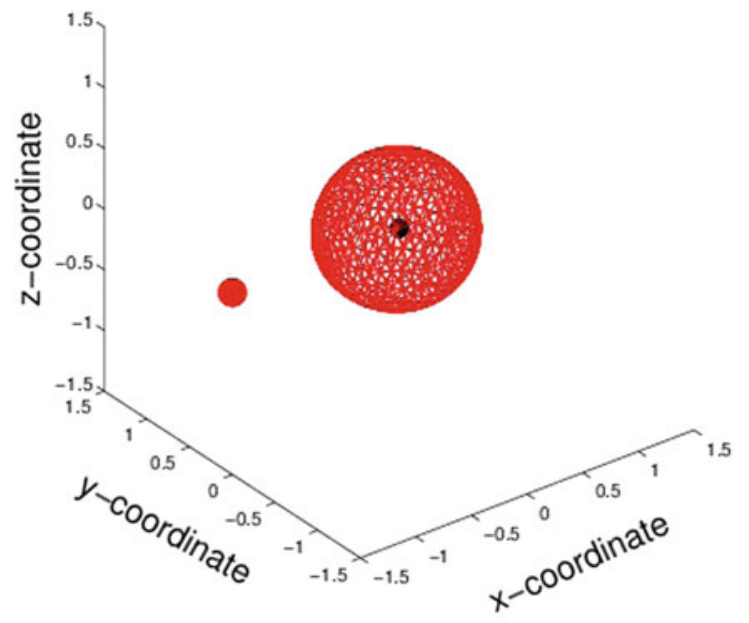

8

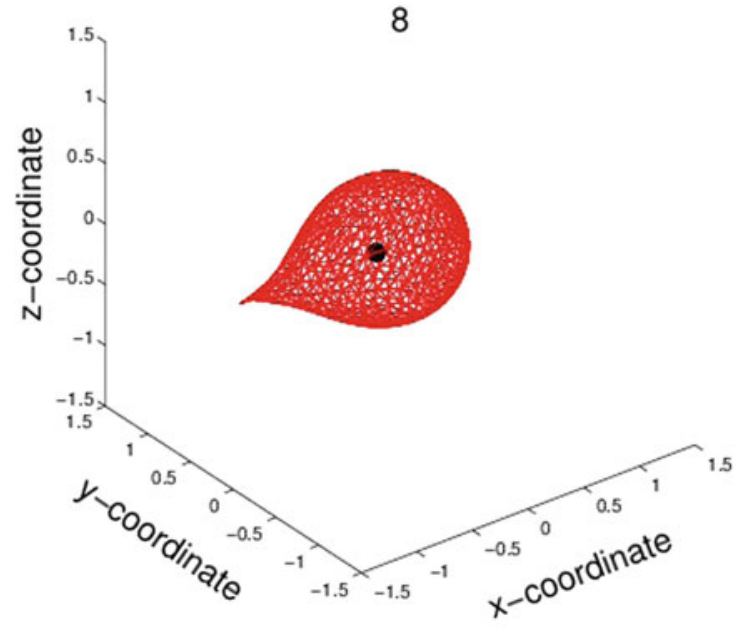

7

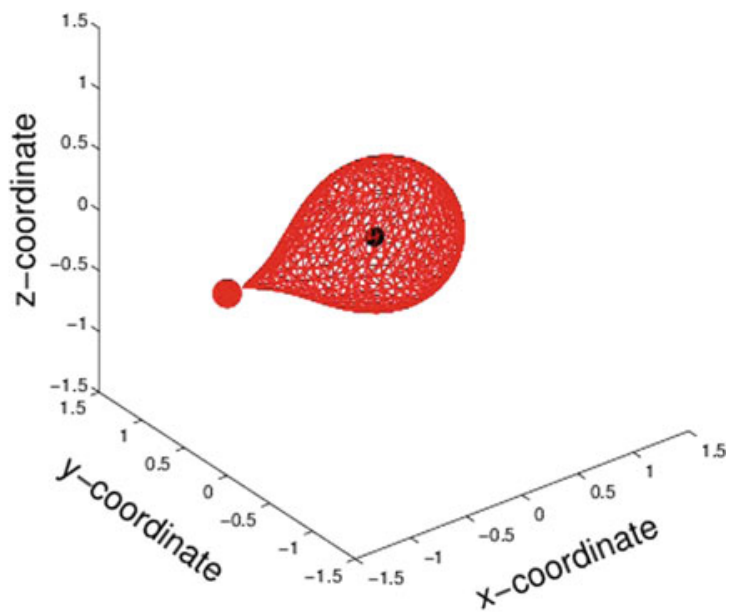

50

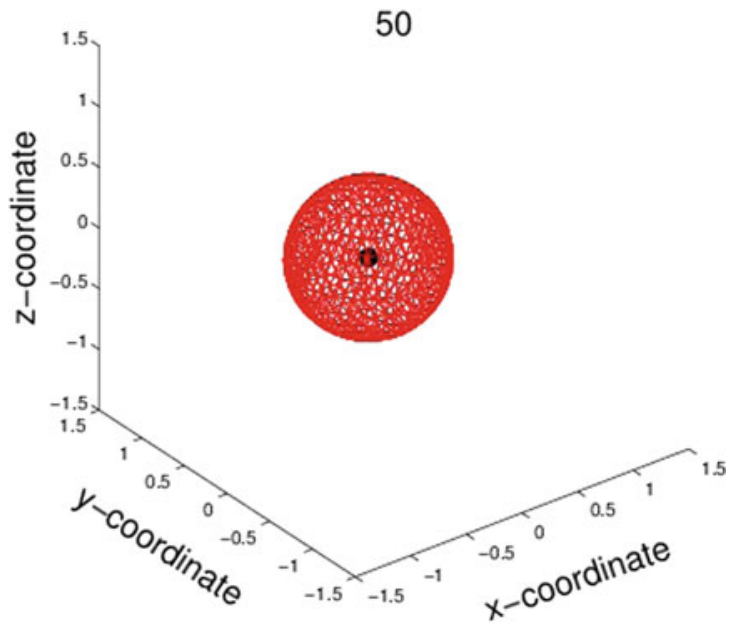

Fig. 13 Snapshots at 1, 7, 8, and 50 time-steps for a 3-D cell exposed to one bacterium

to quantify the sensitivity of the points on the surface with respect to the emitted chemical signal. We expect that the current semi-analytic formalism based on Green's Functions can still be used then. We will investigate this possibility in future studies. We also intend to carry out a mathematical study since we do not exclude the possibility of obtaining analytic solutions. With respect to potential extensions and applications of the current model, we note the following examples. [i] Phagocyte cells that will be allowed to engulf a (moving) point source representing bacteria that secrete chemicals, that is, the cell chases the bacteria until contacting it, and, when the plasma membrane of the cell will circumference a certain percentage of the perimeter of the point source (bacterium), that point source will be deleted from the domain (digested by the phagocyte cell). This will allow simulations of the immune cell response to bacterial (or viral) infections. Here, it is possible to let phagocytes appear by migration through the capillary wall at random locations on the capillary. [ii] Differentiating adipocyte cells that grow in area as internalized lipid droplets synthesized at the cytosol of the cell grow in size and merge together. The mechanism of lipid droplet formation is modeled as pattern formation using Cahn-Hilliard theory (Vermolen et al. 2012). This will require that the (projected) cell areas will be allowed to grow (as cells mature) rather than being kept constant. [iii] Differentiating myoblast cells that fuse together as they contact-thereby creating myofibers which are giant cells that contain a multitude of nuclei. This will again require that cell area would be allowed to grow, but as opposed to adipocytes that tend to become more spherical in shape when maturing, myofibers are elongated; hence, this will require to assume that new myoblasts will be allowed to attach only at certain points to the developing myofibers, in order to produce the elongated shape of the fused cells. [iv] Dendritic cells which are allowed to grow cellular extensions (filapodia or the like) along a gradient of a chemical. This concept can, for example, be employed for simulating development of axons in a neuron. Again, such application will 

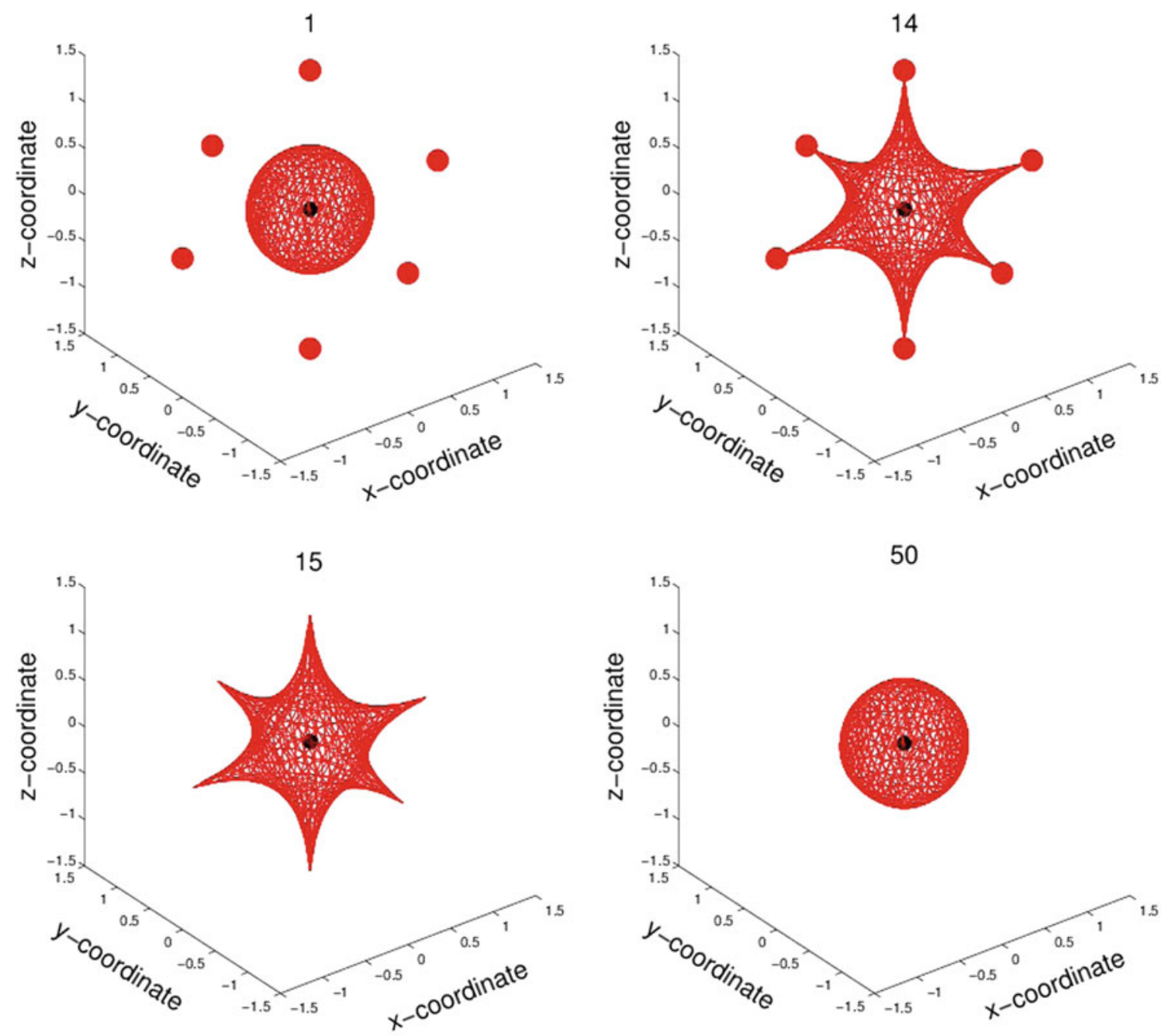

Fig. 14 Snapshots at 1, 14, 15, and 50 time-steps for a 3-D cell exposed to six bacteria

assume that (the projected) cell area is changing with time. [v] Finally, it is possible to consider the use of the present modeling framework for simulating diseases where cell deformability (stiffness) has been abnormally changed, for example, in malaria, sickle cell anemia, or some types of cancer.

Importantly, such model adaptations are straightforward to implement using the present modeling framework, since this essentially requires changing the stiffness of the springs representing the cytoskeleton of the cell. For example, the present modeling can be used to simulate microfluidics experiments where cells with abnormal deformability properties (i.e. high stiffness of the cytoskeleton) are required to propagate along a narrow canal, and then, it could be studied how such cells perform with respect to normal cells. There are direct biological and clinical implications to this problem, for example, red blood cells in patients with sickle cell anemia have difficulty moving in capillaries which then causes serious circulatory problems in this disease. The computation time is expected to remain in the order of several minutes for the single-cell case and in the order of an hour for a multi-cell case in a Matlab programming environment on a $2.66 \mathrm{GHz}$ Intel processor if immediate movies are generated. For the computation of the order of magnitude of the number of floating point operations, we bear in mind that each cell contains $n$ points. These $n$ points have to communicate with $n$ points on $n_{\text {cell }}-1$ cells. Hence, for each cell, the number of floating point operations (flops) is of the order $n \times n \times\left(n_{\text {cell }}-1\right)$. Since this is done for $n_{\text {cell }}$ cells, we see that the total number of flops is of the order $n_{\text {cell }} \times\left(n_{\text {cell }}-1\right) \times n^{2}$. This is regardless of dimensionality, though in three dimensions the number of cells and number of points at the cell surface may be larger than in the two-dimensional case. A large advantage of the present method is that we only need to carry out basic arithmetic operations such as multiplication, division, and additions. To illustrate the dependence of the CPU time on the number of cells, we plot the CPU time versus the number of cells in Fig. 19. The CPU time has been determined 

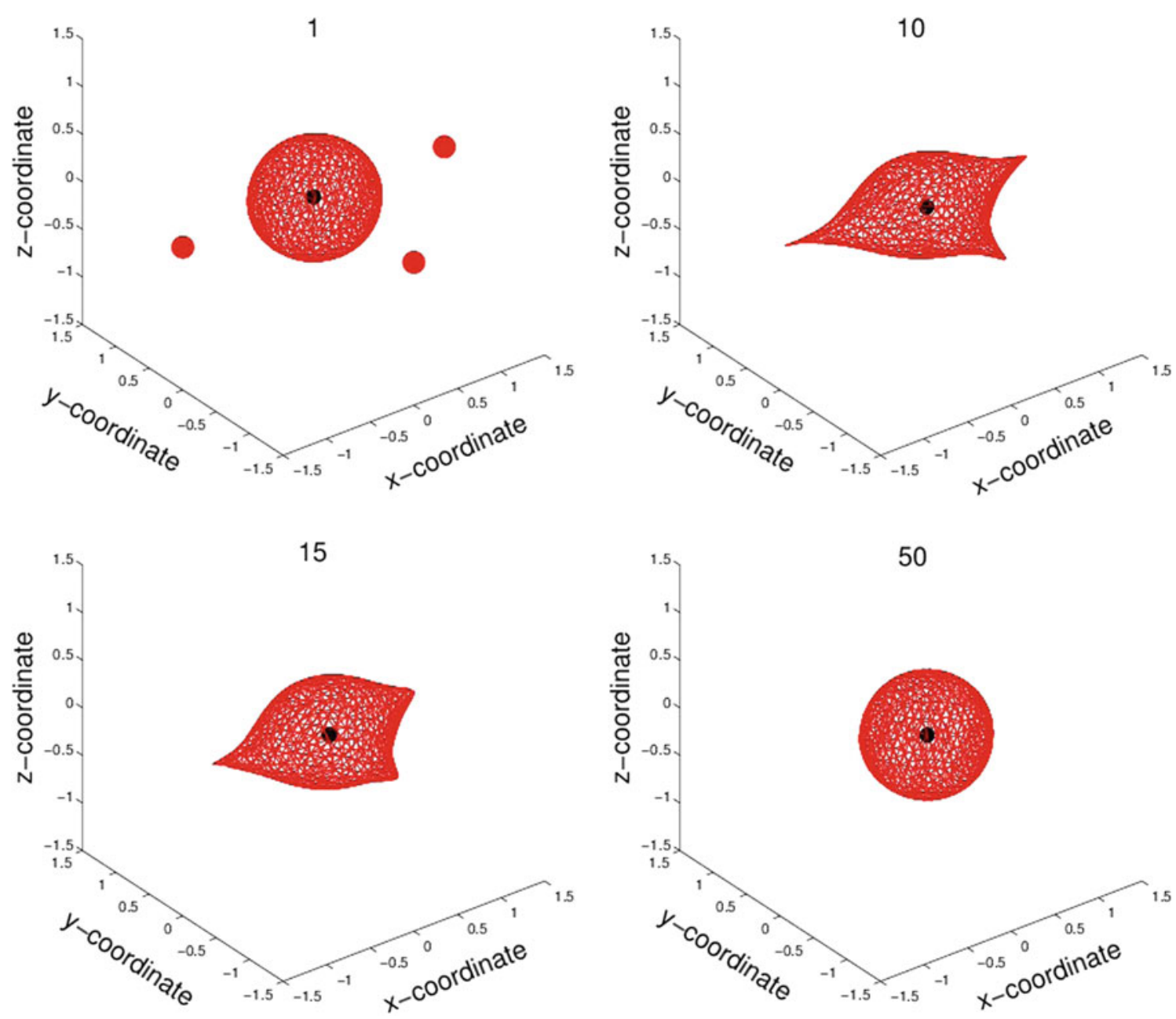

Fig. 15 Snapshots at 1, 10, 15, and 50 time-steps for a 3-D cell exposed to three bacteria

using the Matlab Command 'cputime'. Here, we plot the actual CPU time that involves the displacement of the cell points and the computation of the center of mass per time iteration. This number has been determined as the average CPU time per time iteration over 500 time-steps. Further, post-processing operations, such as plotting the projection of the cells, and the computation of the CSI, cell areas, and circumference are omitted. We use 40 nodes per cell. The figure demonstrates a quadratic behavior as the number of cells increases. Since we postulate that the CPU time depends on the number of grid nodes per cell and the number of cells by

$\lim _{\substack{n_{\text {cell }} \rightarrow \infty \\ n \rightarrow \infty}} \frac{\text { CPUTIME }}{n^{2} n_{\text {cell }}\left(n_{\text {cell }}-1\right)}=\kappa$,

the resulting values of $\kappa$ versus the number of cells are plotted in Fig. 19, and it can be seen that the $\kappa$-value converges toward a constant as the number of cells increases. We think that it is not necessary to use more cells to demonstrate this excellent convergence behavior. For completeness, we performed a computation with 100 cells, having 40 gridnodes on its circumference, and the processing of 100 time-steps took approximately $45 \mathrm{~min}$, which is entirely in line with Eq. (36). We note that the CPU time necessary for the 3-D cell with 50 elements and 588 nodes takes about $0.07 \mathrm{~s}$ per time-step for modeling migration of one cell.

A simple calculation shows that upon having 1,000 cells instead of 7 cells will take about $100 \mathrm{~s}$ per timestep in a sequential computing environment. For the case of hundreds of cells, we want to investigate whether it is possible to use the Graphical Processing Unit (GPU) on the computer as calculating processor. These GPU's are suitable for fast parallel simple arithmetic operations with a very short communication time. In the present calculations, we assumed that each nodal point on the cell surface/circumference secretes a chemical and moves according to the gradient of the chemo-attractant released by all other points on the other cells. The calculation times will fall drastically upon assuming fewer 'active' points 

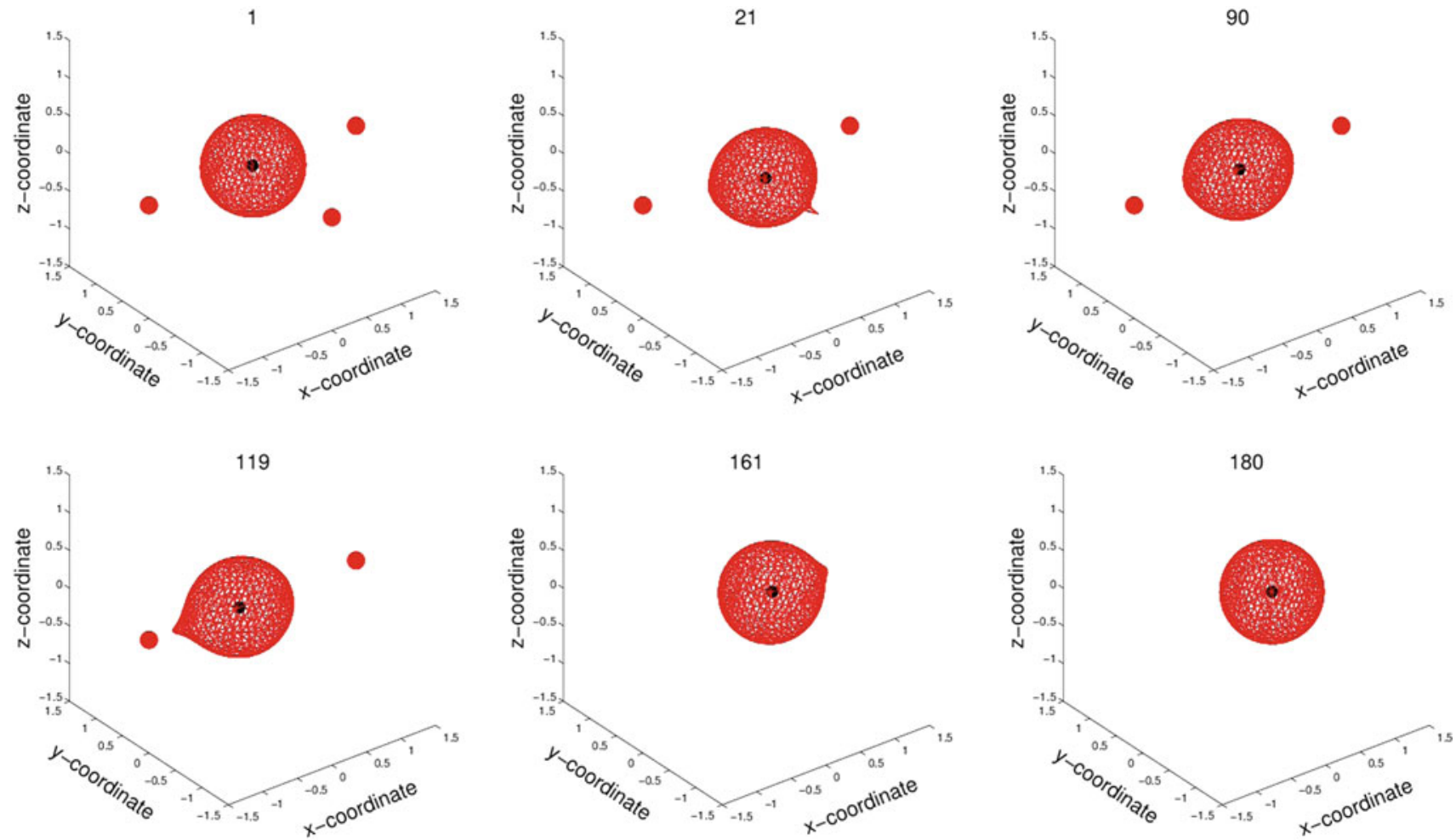

Fig. 16 Snapshots at 1,21, 90,119, 161 and 180 time-steps for a 3-D cell exposed to three bacteria. In this run the spring force $\alpha=150$ has been used, which models a barely deformable cell
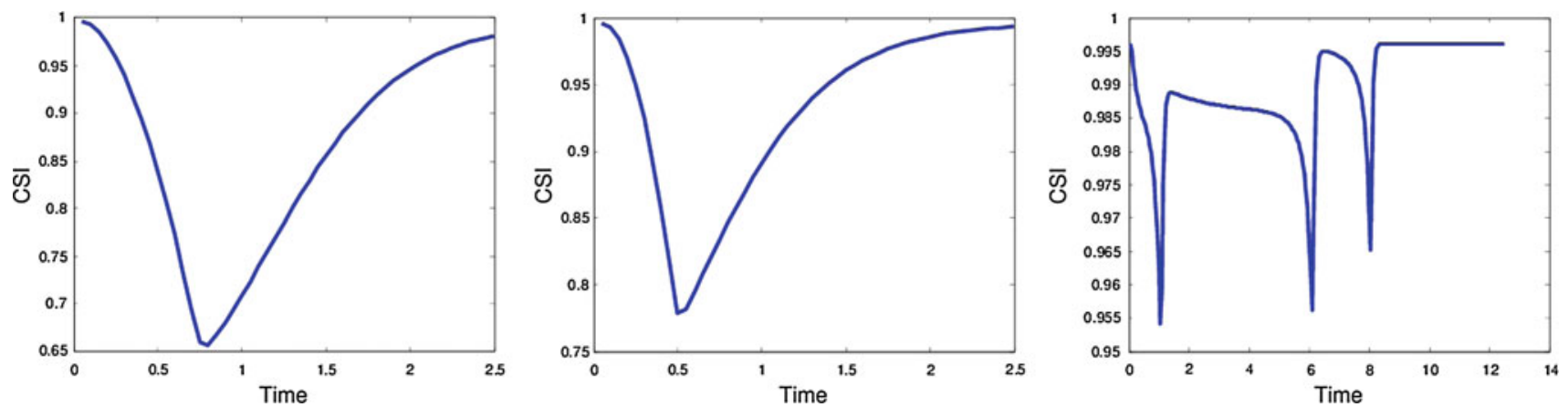

Fig. 17 The CSI versus time for a 3-D cell in the case of 6 sources, see Fig. 14, and for three sources with a low and high cell stiffness, see Figs. 15 and 16 , respectively

on the cell surface/circumference. This issue deserves further elaboration. In the case of solving partial differential equations using for instance finite-element discretization methods, large (nonlinear) systems of algebraic equations have to be solved using Krylov subspace or multigrid methods (or combinations of them), which introduce many more flops.

We further realize that the current Green's Functions that we are using are just approximations for the case of a finite sized computational region, as well as that they are suitable for isotropic and homogeneous media only. However, if the computational domain is assumed to be large compared (as we assume) with the cell sizes and intercellular distances, then this approximation is very accurate. For finite domains, one can use specified Green's Functions; however, the disadvantage is that we end up with infinite series that have to be truncated anyway. Hence, our current approach could be considered as computationally elegant due to the single-term nature of the analytic solutions that we use.

We further note that we disregarded tangential forces in the current model. Surface tension of the cell is solely modeled by the driving force which lets the cell return to circular or spherical geometry in the case of absence of chemical attractors or repellors. The incorporation of tangential forces 

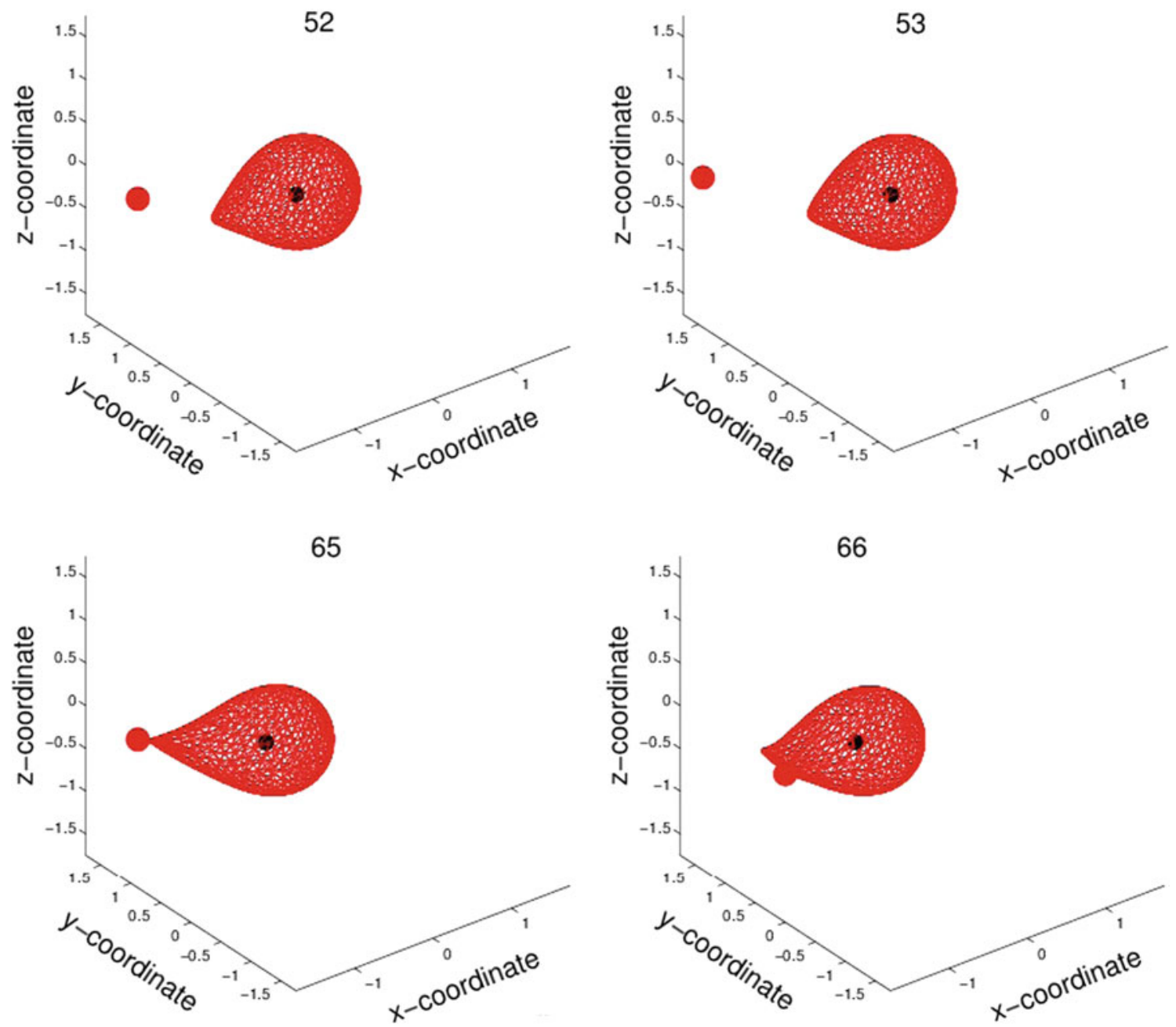

Fig. 18 Snapshots at 52, 53, 65, and 66 time-steps for a 3-D cell exposed to one moving bacterium using the transient Green's Functions for the chemical secreted by the bacterium
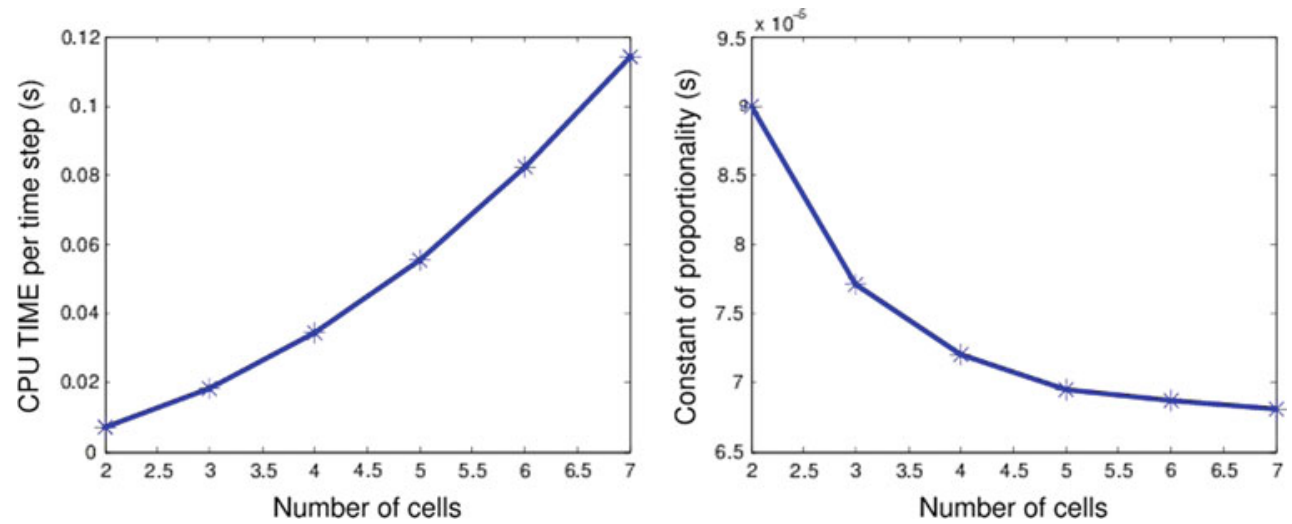

Fig. 19 Left: the CPU Time in seconds per time iteration as a function of the number of cells. Right: convergence to a power law, with $\kappa$, upon increasing the number of cells

into the model is interesting; however, it would make the model inherit some properties of solving partial differential equations on the circumference or surface of the cell. This would increase the computational cost of the current calculations, and therefore, in this preliminary version of the model, we choose to omit this extension in the current study. 

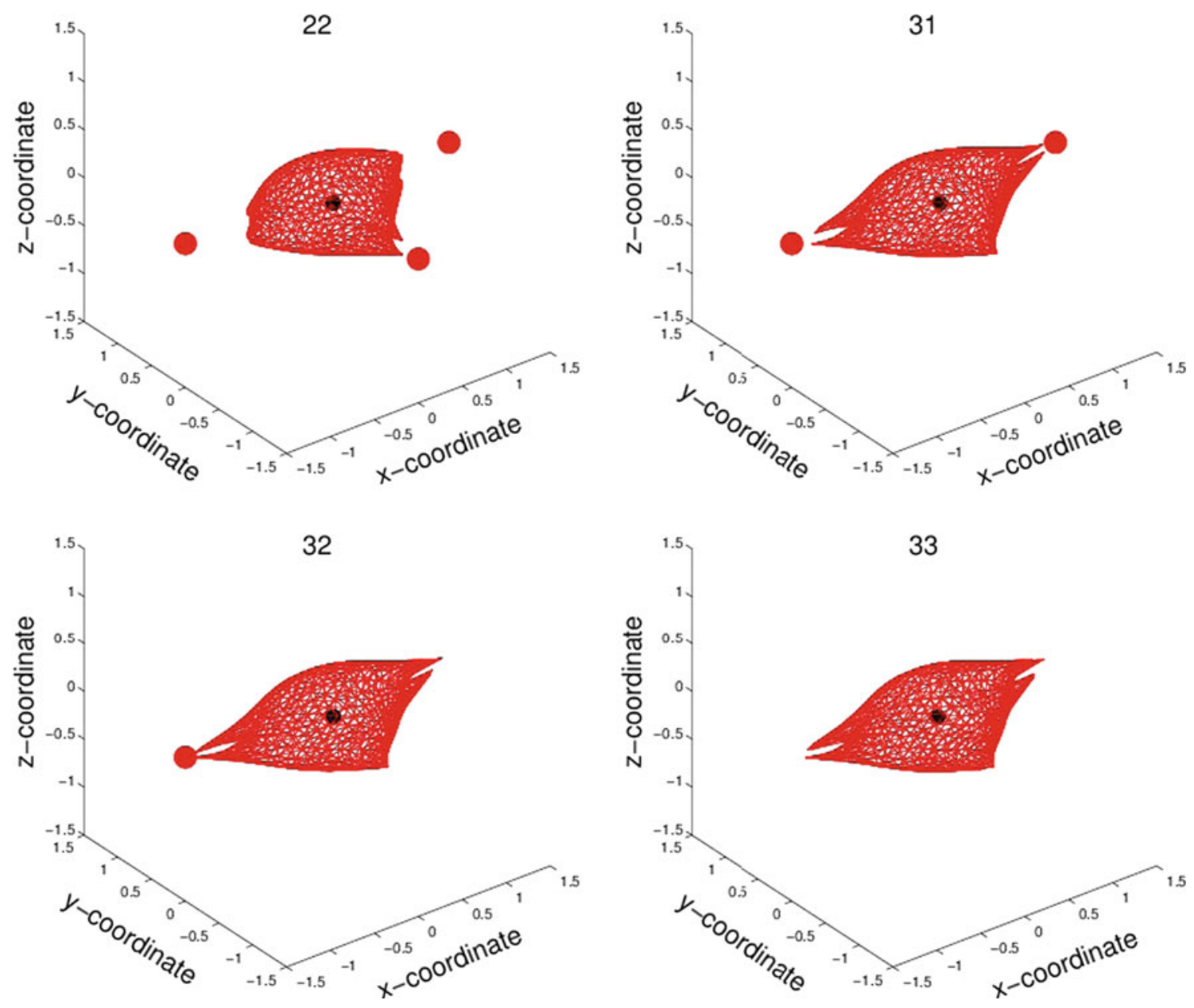

Fig. 20 Snapshots at 22, 31, 32, and 33 time-steps for a 3-D polarized cell exposed to three bacteria. Here $\varepsilon_{P}=0.3$ has been taken

Another reason is that we do not expect to see many qualitative changes since the tangential contributions will also give convergence to the circular/spherical equilibrium cell geometry.

We also note that we assumed that each point of the cell circumference or surface represents a source of the secreted chemical and moves according to the concentration gradient. This is a simplification that can be changed in a straightforward way. However, the net result of just 'choosing' a limited set of points as emitters of the chemical is not going to change the kinetics of the migration of the other cells toward the present cell that much, since motion is directed toward the gradient of the chemical. In both cases, the overall concentration profile obtained from a superposition of all sources does not differ much as long as the overall strength of all sources is the same. Only, if the diffusion constant has a very small value with respect to the speed of the moving sources, then the difference is possibly more significant. Further, it is noted that the polarization of the cell is of importance and that therefore the sensitivity at each point on the cell surface is not constant. To model this polarity, we performed a run with an initially spherical cell on which the sensitivity increases at the poles (the south and north poles) of the cell. In Fig. 20, we show some snapshots at various times for a spherical cell that is exposed to three bacterial sources. The polarization is modeled by the following function

$P(z)=\frac{1+\varepsilon P}{2}\left(1+\frac{1}{2} \cos \left(\frac{2 \pi}{1.2}(z-0.6)\right)\right)$

which is executed on the initial positions of the points on the cell surface. The above function is multiplied by the actual velocity of each point of the cell surface. Here, $\varepsilon_{P}$ determines the amount of polarization. It can be seen in Fig. 20 that the behavior of the cell surface behaves completely differently from the non-polarized case. The simulations were done for a spherical cell exposed to three bacteria. Now the cell consumes the bacteria in a more sequential order, and furthermore, the cell reaches the bacteria by using two protrusions. We also plot the CSI as a function of time for different levels of perturbations, in Fig. 21. Here, it can be seen that the behavior of the CSI versus time changes drastically since the 


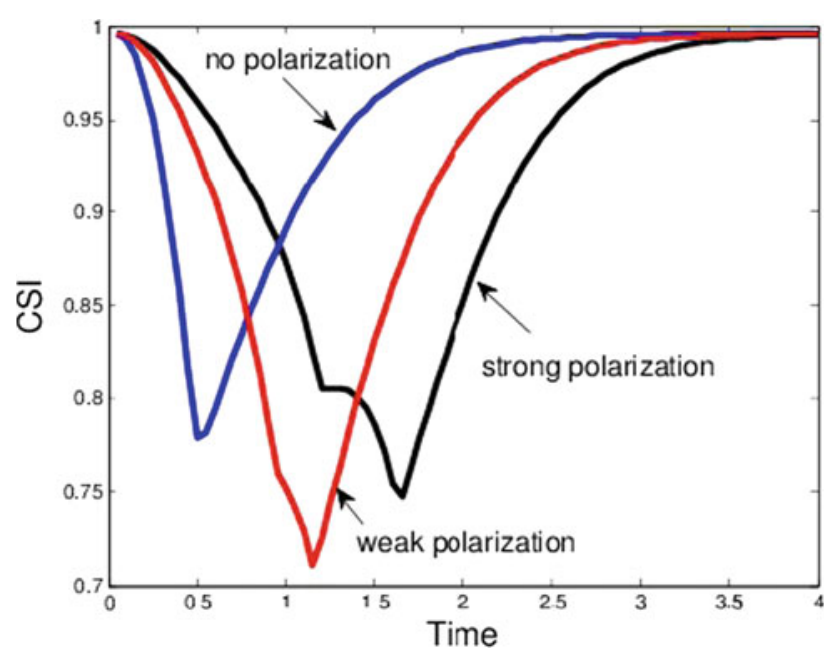

Fig. 21 The CSI as a function of time for three different levels of polarization $\left(\varepsilon_{P}=0,0.3,0.6\right)$ of the initially spherical cell exposed to three bacterial sources
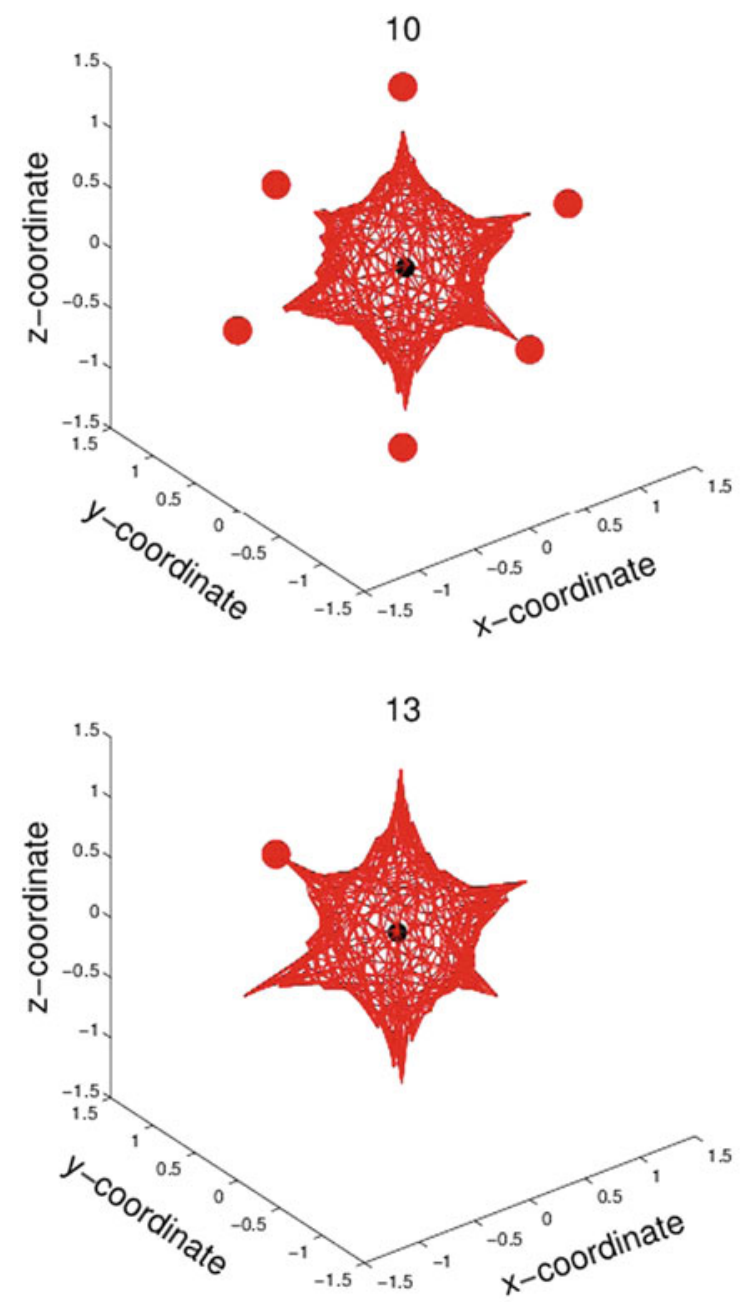

points at the poles of the cells are the points that move most rapidly.

Finally, the model uses Green's Functions for the concentration profiles, and hence, the model is based on homogeneity and isotropic conditions for the medium through which diffusion takes place. In order to qualitatively model anisotropies and heterogeneities, we include a random component into the rate of motion of each point on the cell surface by adapting the velocity at each point on the cell surface by

$\beta \longrightarrow \beta(1+\varepsilon * \operatorname{rand}(p))$,

where $\operatorname{rand}(p)$ is a stochastic function for which $\operatorname{rand}(p)$ : $\{1, \ldots, n\} \rightarrow[-1,1]$. The results in terms of snapshots at various times for a cell with six bacteria are shown in Fig. 22. The times at which the bacteria are consumed are not the same any longer. It can also be seen that the cell looks less smooth than in the non-randomly perturbed case. Further, we plot the CSI as a function of time for various degrees of randomness
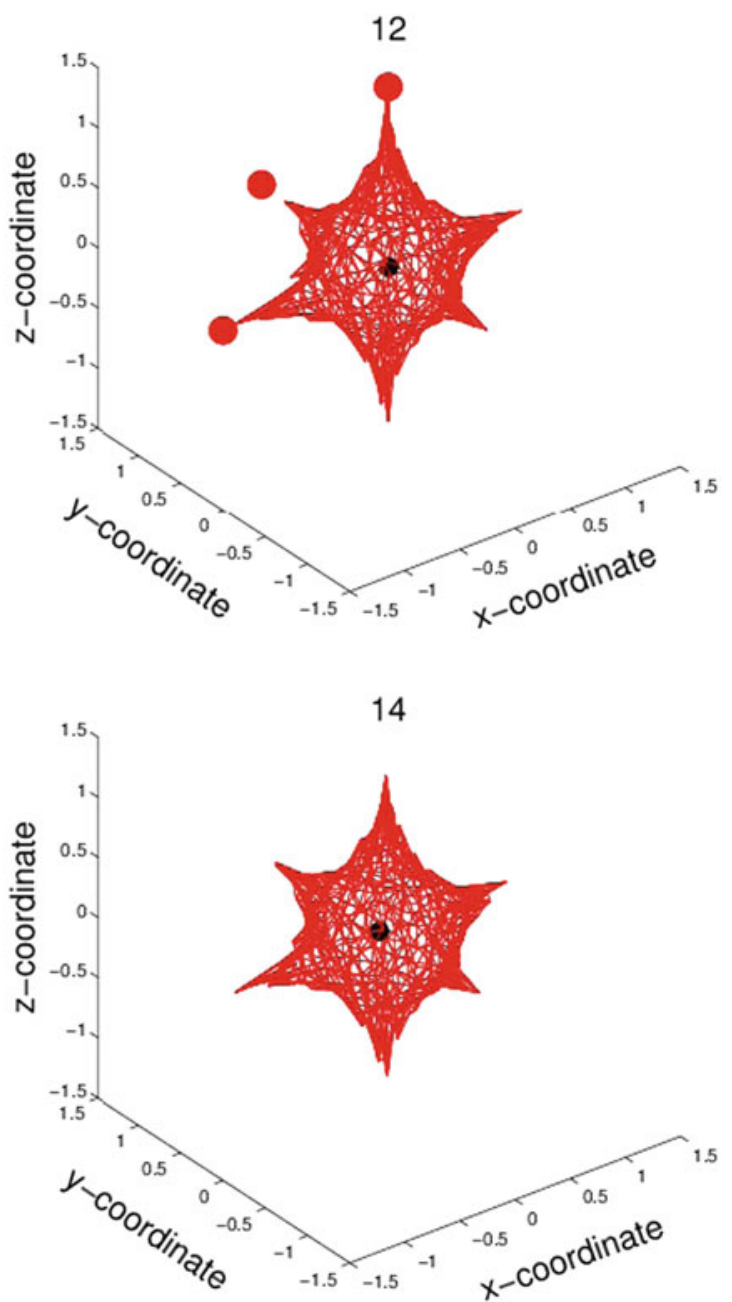

Fig. 22 Snapshots at 1, 10, 15, and 50 time-steps for a 3-D cell exposed to six bacteria subject to a random component in the motion, as modeled in Eq. (38) 


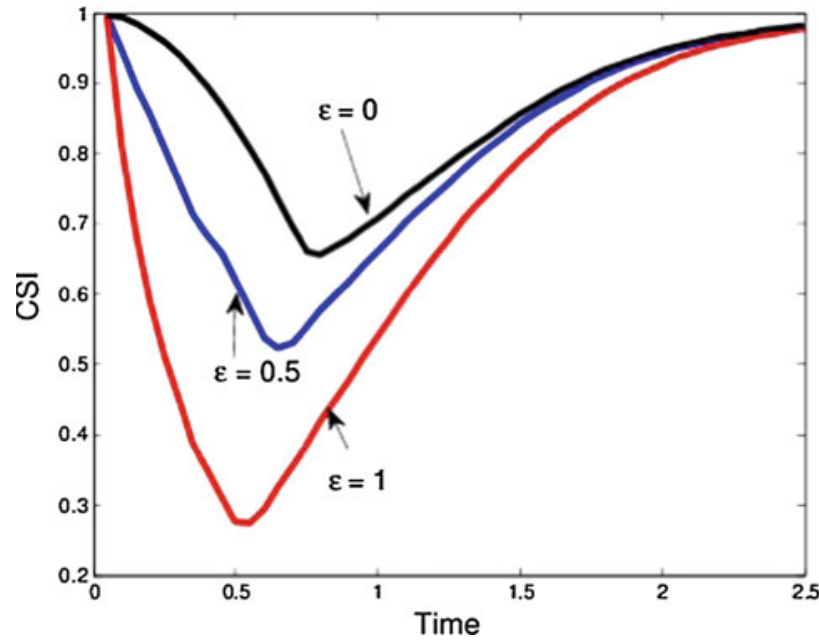

Fig. 23 The CSI as a function of time for three different levels of randomness in the motion of the points on the surface of the initially spherical cell exposed to three bacterial sources. The randomness is taken into account by use of Eq. (38)

in Fig. 23. The plots hardly change from a qualitative point of view; however, the imposed randomness makes the CSI fall more significantly, as expected since the deviations from the smooth spherical shape are larger.

In order to document changes in cell shape using a single experimental design, it is convenient to use a wound healing assay, where cells migrate, attach, spread and grow in density up to confluency over a time course of about a day. To demonstrate qualitative similarities between the present theoretical predictions and empirically-observed cell shapes, we use the wound healing protocol which has been described in a previous publication (Topman et al. 2011) and is summarized here for completeness. Fibroblasts of the NIH3T3 line were cultured in a growth medium (GM) composed of Dulbecco's modified Eagle Medium (DMEM) with 4.5g/l D-glucose, $10 \%$ fetal bovine serum, $2 \mathrm{mM}$ glutamine, 0.1 $\mu \mathrm{g} / \mathrm{ml}$ penicillin and $0.1 \mu \mathrm{g} / \mathrm{ml}$ streptomycin (Biological Industries Co., Israel). Cells were first thawed from liquid nitrogen storage and cultured at $37{ }^{\circ} \mathrm{C}$ and $5 \% \mathrm{CO}_{2}$ until cultures were nearly confluent. Then, cells were passaged by washing the cultures twice with PBS, adding $1 \mathrm{ml}$ of trypsin-EDTA $(0.25 \% / 0.25 \%)$ for $2-5 \mathrm{~min}$, adding $2 \mathrm{ml}$ of $\mathrm{GM}$, centrifuging at $300 \mathrm{~g}$ for $7 \mathrm{~min}$ and removing the supernatant. Cells were then re-cultured (in 6-well plates), again to near confluence, following which local damage was inflicted to the cultures by means of an approximately $400 \mu \mathrm{m}$-wide micro-indentor which compressed the monolayers locally. Digital contrast phase microscopy micrographs were captured (using an Eclipse TS100 Nikon microscope connected to a DS-Fi1 Nikon camera) every $2 \mathrm{~h}$ with the $\times 10$ objective. The resolution of the captured micrographs was 2,560 $\times 1,920$ ( 3 pixels/micron) and the digital field of view was

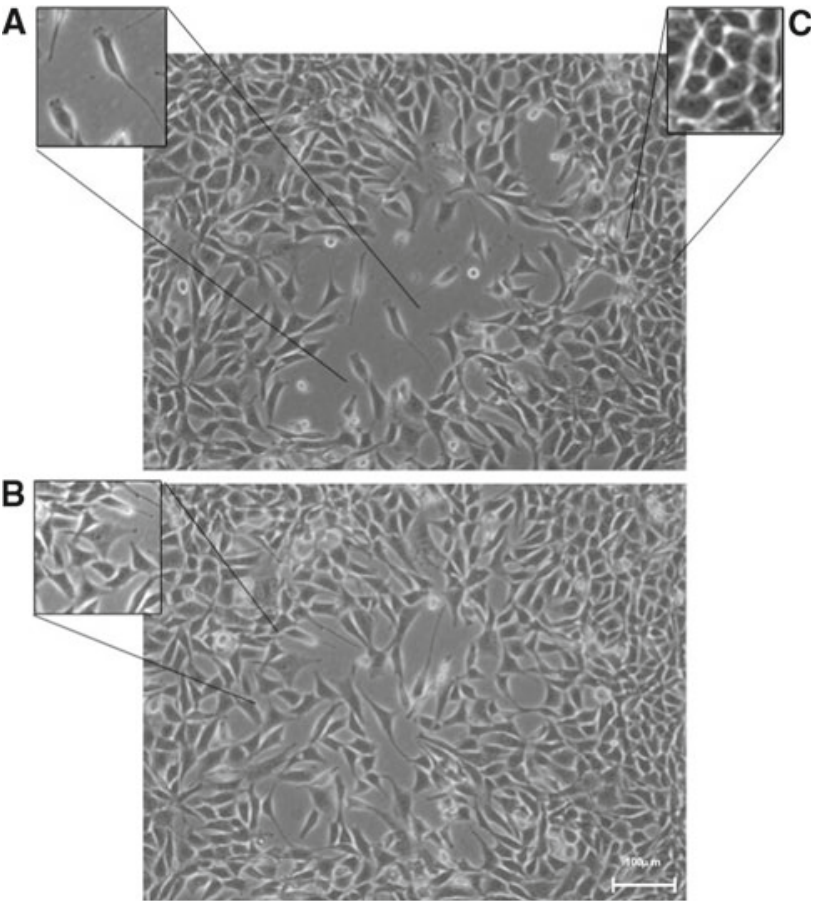

Fig. 24 Two time-sequence micrographs, taken $4 \mathrm{~h}$ apart, which demonstrate shape changes in NIH3T3 fibroblasts which cover a local damage site: $(A)$ Oval elongated shape of migrating cells. $(B)$ multi-polar cell shapes when cells are resting and well-spread at a sub-confluent density; note the 4-poles cell in the center of the magnified frame. $(C)$ Polygonal cell shapes in dense confluent sites. The scale-bar represents $100 \mu \mathrm{m}$

$850 \times 640 \mu \mathrm{m}$. Cell shapes were documented through the process of coverage of the local damage site by the migrating cells. Figure 24 shows two time-sequence micrographs, taken $4 \mathrm{~h}$ apart. These nicely demonstrate the typical shapes and shape changes in the NIH3T3 fibroblasts in the process of coverage of the local damage site. Frame A shows migrating cells that take the typical oval elongated shape. Frame B shows multi-polar cell shapes that are typically observed when cells are resting and well-spread at a sub-confluent density. Note the 4-poles cell in the center of the magnified frame, which resembles the case of a single cell surrounded by four source points which has been analyzed theoretically herein. Frame $\mathrm{C}$ shows the cobblestone-shaped structures formed in fully confluent sites, with the individual cells taking shapes of polygons. Hence, the cell shapes captured in the wound healing assay experiment in Fig. 11 nicely demonstrate the entire span of cell shapes produced by the present modeling. Specifically, oval elongated cell shapes of migrating cells were produced in the simulations in Figs. 5 and 7; multi-polar cell shapes were generated in the simulations shown in Fig. 3; and cobblestone-shaped cells in dense cultures were depicted in the simulations presented in Figs. 8 and 9. The modeling is therefore versatile in simulating all these possible planar cell shapes. 


\section{Conclusions}

A model for the displacement and deformation of cells has been constructed on the basis of chemotaxis. The concentration gradients are obtained from Green's Functions for the solution of the (quasi steady-state) diffusion equations with (multiple) source terms. The model is extended to a case of a small multi-cell colony, in which the simulated cellular motion qualitatively agrees with experimental observations. Further, as a result of the computational simplicity of the model, the computation times are very small, also for three-dimensional configurations. Some simulations are shown, both in two and three spatial dimensions, under various circumstances, such as polarization, heterogeneities, and cell stiffness. Further, for fast moving chemical point sources near the cell, a retardation effect for the direction of migration of the cell in the simulations is observed if timedependent Green's Functions are used.

Acknowledgment The manuscript benefitted from the revisions that were suggested by the referees.

Open Access This article is distributed under the terms of the Creative Commons Attribution License which permits any use, distribution, and reproduction in any medium, provided the original author(s) and the source are credited.

\section{References}

Adam JA (1999) A simplified model of wound healing (with particular reference to the critical size defect). Math Comput Model 30: 23-32

Arciero J, Mi Q, Branca MF, Hackam DJ, Swigon D (2011) Continuum model of collective cell migration in wound healing and colony expansion. Biophys J. doi:10.1016/j.bpj.2010.11.083

Armstrong NJ, Painter KJ, Sherratt JA (2006) A continuum approach to modeling cell-cell adhesion. J Theor Biol 243:98-113

Atkins P, de Paula J (2009) Atkins' Physical Chemistry, 9th edn, Chap. 18. Oxford University Press, Oxford

Axelrod JD (2006) Cell shape in proliferating epithelia: a multifaceted problem. Cell 126:643-645

Baker RE, Gaffney EA, Maini PK (2008) Partial differential equations for self-organization in cellular and developmental biology. Nonlinearity 21:R251-R290

Barreira R, Elliott CM, Madzvamuse A (2011) The surface finite-element method for pattern formation on evolving biological surfaces. J Math Biol 63:1095-1119

Evans LC (1998) Partial differential equations. Americal Mathematical Society, Providence

Gaffney EA, Pugh K, Maini PK (2002) Investigating a simple model for cutaneous wound healing angiogenesis. J Math Biol 45(4): 337-374

Gerisch A, Chaplain MAJ (2006) Robust numerical methods for taxisdiffusion-reaction systems: Applications to biomedical problems. Math Comput Model 43:49-75

Geris L, Gerisch A, Schugart RC (2010) Mathematical modeling in wound healing: bone regeneration and tissue engineering. Acta Biotheor 58(4):355-367
Geris L, Vandersloten J, Van Oosterwyck H (2009) In silico biology of bone modelling and remodelling: regeneration. Phil Trans R Soc A 367:2031-2053

Graner F, Glazier J (1992) Simulation of biological cell sorting using a two-dimensional extended Potts model. Phys Rev Lett 69:20132016

Gray BL, Lieu DK, Collins SD, Smith RL, Barakat AI (2002) Microchannel platform for the study of endothelial cell shape and function. Biomed Microdev 4(1):9-16

Javierre E, Moreo P, Doblaré M, García-Aznar, MJ (2009) Computational Modelling of wound contraction, In: Proc Congreso de Métodos Numéricos en Ingeniería 2009, Barcelona, SEMNI

Javierre E, Vermolen FJ, Vuik C, Zwaag Svan der (2009) A mathematical analysis of physiological and morphological aspects of wound closure. J Math Biol 59:605-630

Lin X, Helmke BP (2009) Cell structure controls endothelial cell migration under fluid shear stress. Cell Mol Bioeng 2(2):231-243

Merks MH, Koolwijk P (2009) Modeling morphogenesis in silico and in vitro: towards quantitative, predictive, cell-based modeling. Math Mod Nat Phenom 4(4):149-171

Mi Q, Swigon D, Riviere B, Cetin S, Vodorotz Y, Hackam D (2007) One-dimensional elastic continuum model of enterocyte layer migration. Biophys J 93:3745-3752

Murray JD (2004) Mathematical biology II: spatial models and biomedical applications. Springer, New York

Neilson MP, MacKenzie JA, Webb SD, Insall RH (2011) Modeling cell movement and chemotaxis using pseudopod-based feedback. SIAM J Sci Comput 33(3):1035-1057

Olsen L, Sherratt JA, Maini PK (1995) A mechanochemical model for adult dermal wound closure and the permanence of the contracted tissue displacement role. J Theor Biol 177:113-128

Schugart RC, Friedman A, Zhao R, Sen CK (2008) Wound angiogenesis as a function of tissue oxygen tension: a mathematical model. Proc Natl Acad Sci USA 105(7):2628-2633

Segal A (2012) SEPRAN manuals, Den Haag, 1982-2012.

Sherratt JA, Murray JD (1991) Mathematical analysis of a basic model for epidermal wound healing. J Math Biol 29:389-404

Topman G, Sharabani-Yosef O, Gefen A (2011) A standardized objective method for continuously measuring the kinetics of cultures covering a mechanically damaged site. Med Eng Phys. doi:10. 1016/j.medengphy.2011.07.014

Vermolen FJ (2009) A simplified finite-element model for tissue regeneration with angiogenesis. ASCE J Eng Mech 135(5):450-460

Vermolen FJ, Gefen A (2012) A semi-stochastic cell-based formalism to model the dynamics of migration of cells in colonies. Biomech Model Mechanobiol 11(1-2):183-195

Vermolen FJ, Javierre E (2009) On the construction of analytic solutions for a diffusion-reaction equation with a discontinuous switch mechanism. J Comput Appl Math 231:983-1003

Vermolen FJ, Javierre E (2010) Computer simulations from a finiteelement model for wound contraction and closure. J Tissue Viability 19:43-53

Vermolen FJ, Javierre E (2011) A finite-element model for healing of cutaneous wounds combining contraction angiogenesis and closure. J Math Biol. doi:10.1007/s00285-011-0487-4

Vermolen FJ, Segal A, Gefen A (2011) A pilot study of a phenomenological model of adipogenesis in maturing adipocytes using Cahn-Hilliard theory. Med Biol Eng Comput 49(12): 447-457

Xue C, Friedman A, Sen CK (2009) A mathematical model of ischemic cutaneous wounds. Proc Natl Acad Sci USA 106(39): 16783-16787 